\title{
An Application of CFD to Guide Forced Boundary-Layer Transition for Low-Speed Tests of a Hybrid Wing-Body Configuration
}

\author{
James M. Luckring ${ }^{1}$, Karen A. Deere ${ }^{2}$ \\ NASA Langley Research Center, Hampton, VA, 23681 \\ Robert E. Childs ${ }^{3}$, Paul M. Stremel ${ }^{4}$ \\ Science and Technology Corporation, Moffett Field, CA, 94035 \\ Kurtis R. Long ${ }^{5}$ \\ NASA Ames Research Center, Moffett Field, CA, 94035
}

\begin{abstract}
A hybrid transition trip-dot sizing and placement test technique was developed in support of recent experimental research on a hybrid wing-body configuration under study for the NASA Environmentally Responsible Aviation project. The approach combines traditional methods with Computational Fluid Dynamics. The application had threedimensional boundary layers that were simulated with either fully turbulent or transitional flow models using established Reynolds-Averaged Navier-Stokes methods. Trip strip effectiveness was verified experimentally using infrared thermography during a low-speed wind tunnel test. Although the work was performed on one specific configuration, the process was based on fundamental flow physics and could be applicable to other configurations.
\end{abstract}

\begin{tabular}{|c|c|c|c|}
\hline \multicolumn{4}{|c|}{ Nomenclature } \\
\hline$A v$ & data average over an interval & $s$ & distance along a surface \\
\hline$b_{1}-b_{3}$ & spanwise coordinate for leading-edge sweep break & $U_{\infty}$ & free-stream reference velocity \\
\hline$C_{f}$ & skin friction coefficient & $x, y, z$ & body-axis Cartesian coordinates \\
\hline$C_{p}$ & pressure coefficient & $u, v, w$ & Cartesian velocity components \\
\hline$c$ & wing chord & & \\
\hline$c_{r e f}$ & reference chord & $\alpha$ & angle of attack, deg. \\
\hline$H_{12}$ & boundary-layer shape factor, $\delta^{*} / \theta$ & $\delta$ & boundary layer thickness \\
\hline$k$ & roughness height & $\delta^{*}$ & boundary layer displacement thickness \\
\hline$M$ & Mach number & $\theta$ & boundary layer momentum thickness \\
\hline$R a$ & data range (maximum-minimum) over an interval & $\Lambda$ & leading-edge sweep angle, deg. \\
\hline $\operatorname{Re}_{\text {cref }}$ & reference chord Reynolds number, $U_{\infty} c_{r e f} / v$ & $\mu$ & viscosity \\
\hline$R e_{k}$ & roughness height Reynolds number, $u_{k} k / v_{k}$ & $v$ & kinematic viscosity, $\mu / \rho$ \\
\hline$R e_{x}$ & length Reynolds number, $U_{\infty} x / v$ & $\rho$ & density \\
\hline $\begin{array}{l}\operatorname{Re}_{\theta} \\
\text { Subscr }\end{array}$ & momentum thickness Reynolds number, $U_{e} \theta / v$ & & \\
\hline$e$ & value at edge of boundary layer & $\infty$ & free-stream reference conditions \\
\hline$k$ & value at roughness height & & \\
\hline
\end{tabular}

\footnotetext{
${ }^{1}$ Senior Research Engineer, Configuration Aerodynamics Branch, james.m.luckring@nasa.gov, AIAA Associate Fellow.

${ }^{2}$ Aerospace Engineer, Configuration Aerodynamics Branch, karen.a.deere@nasa.gov, AIAA Senior Member.

${ }^{3}$ Aerospace Engineer, robert.e.childs@ nasa.gov.

${ }^{4}$ Aerospace Engineer, paul.m.stremel@ nasa.gov.

${ }^{5}$ Senior Research Engineer, Experimental Aerophysics Branch, kurtis.r.long@nasa.gov.
} 
Abbreviations

AEDC Arnold Engineering Development Complex

CFD Computational Fluid Dynamics

ERA Environmentally Responsible Aviation

HWB Hybrid Wing Body

LaRC NASA Langley Research Center
LM Langtry-Menter transitional turbulence model

NASA National Aeronautics and Space Administration

PAI Propulsion Airframe Integration

RANS Reynolds Averaged Navier Stokes

SA Spalart-Allmaras turbulence model

\section{Introduction}

$\mathrm{H}$ ybrid Wing-Body (HWB) concepts have received attention in recent years as one approach to simultaneously address multiple performance metrics for advanced transonic transports such as reductions in fuel consumption, emissions, and noise. The Environmentally Responsible Aviation project (ERA) was established between NASA and Boeing to pursue the development of a hybrid wing-body concept targeted at these often conflicting performance metrics. A representative artist's sketch of the HWB concept is shown in Figure 1. Some of the key features for this concept include a highly swept and thick inner portion of the configuration (the body), which blends with a more conventionally swept transport wing for the outer portion of the configuration. Highbypass-ratio nacelles are shown mounted on the aft upper surface of the body with canted vertical tails at the aft body deck; this arrangement provides for acoustic shielding of the engines.

The HWB represents a radical departure from conventional commercial transports, and as such presents a number of challenges for many of the configuration development disciplines, including aerodynamics. Both transonic performance and low-speed takeoff/landing characteristics offer new and possibly unique challenges (e.g., shock wave and flow separation management, Propulsion-Airframe Integration (PAI)) as compared to the experience from many decades of conventional commercial transport development programs. Both advanced Computational Fluid Dynamics (CFD) and wind tunnel testing can help address these challenges.

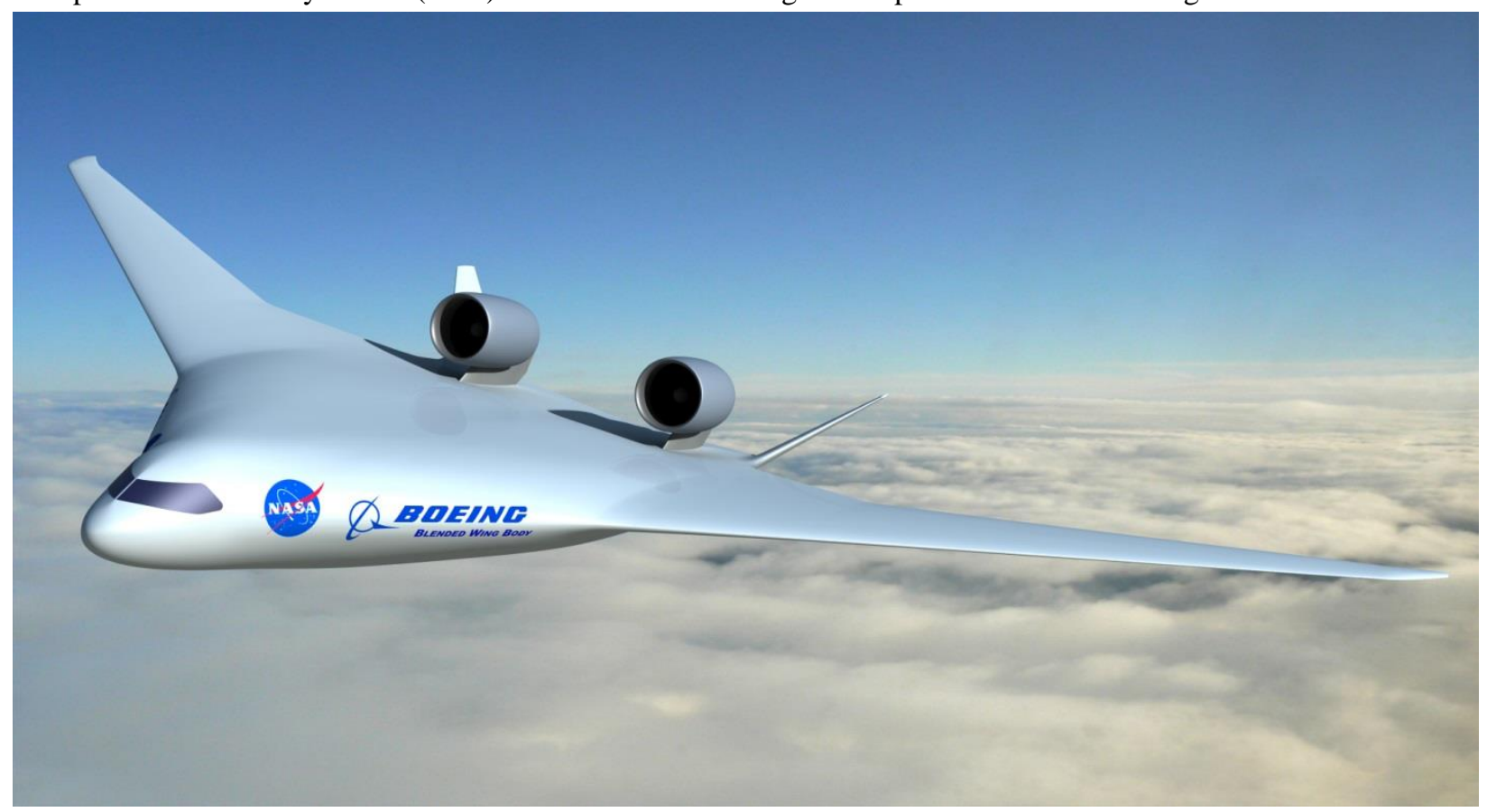

Figure 1. Boeing/NASA hybrid wing-body concept.

The present work was performed in support of low-speed wind tunnel testing in the ERA project. The test program itself was designed to address low-speed configuration aerodynamics of the HWB at takeoff and landing conditions, and these included typical high angles of attack and sideslip that can result in separated flow. Effective tripping of the wind tunnel model boundary layers can, thus, be important to assure that turbulent flow is achieved over most of the model at the wind tunnel test conditions. Without such tripping, the resultant laminar or transitional flows on the wind tunnel model could result in measurements that do not represent full-scale flows. 
One particular concern was for effective tripping of the boundary layers that traverse the highly-swept and blunt inboard portion of the hybrid wing-body configuration. Flow over the body upper surface proceeds toward the engines, and any separated flow from the body could have important PAI consequences. An example is shown in Figure 2 from a CFD simulation of the turbulent flow about the Hybrid-Wing-Body configuration of the present study at wind tunnel test conditions. The configuration includes a deployed leading-edge slat. The simulation was performed

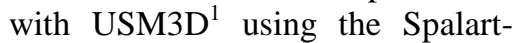
Allmaras turbulence model, ${ }^{2}$ and shows surface streamlines as well as crossflow plane contours of total pressure loss. The angle of attack is high but within the planned test program. Despite this high angle of attack, the simulation shows attached flow about the blunt and highly-swept leading edge of the body. Further downstream smoothsurface flow separation is predicted on the upper surface of the body. The details of this flow are complex, and probably not fully understood, but for the wind tunnel test to provide estimates that are

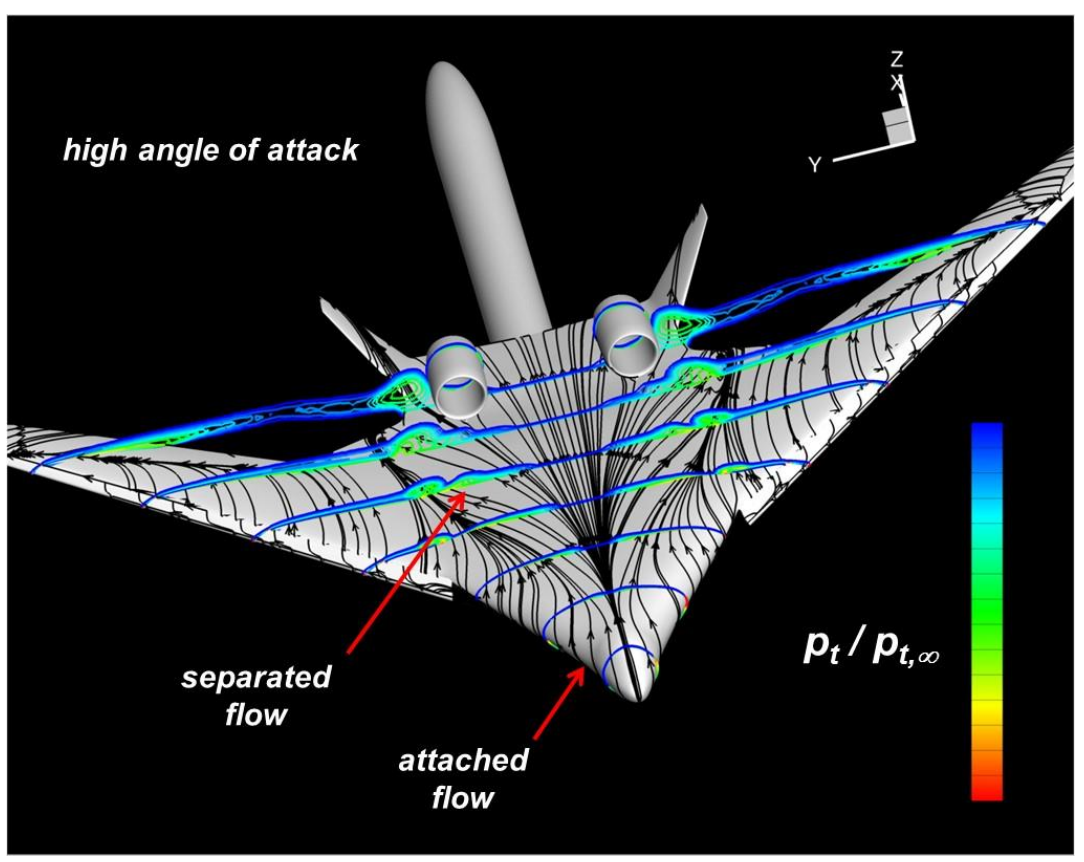

Figure 2. Turbulent CFD simulation of HWB flow at high angle of attack and wind tunnel test conditions. $M=0.2, R_{\text {cref }}=5.27 \times 10^{6}$. relevant to full-scale vehicle characteristics, it is important that the flow about the model be governed by turbulent flow physics. For groundbased testing, this then necessitates effective tripping of the boundary layers to create turbulent flow on the wind tunnel model.

The highly-swept and blunt leading edge of the body differs significantly from many previous testing experiences, and CFD thus became an attractive consideration, in conjunction with traditional methods, ${ }^{3,4}$ to guide the placement and sizing of boundary layer trips. However, caution was also needed since CFD predictions with transitional flows are not well anchored, and a hybrid approach was adopted that combined physics-based reasoning from traditional techniques with flowfield simulations from several CFD methods with different boundary-layer simulations. Emphasis was placed on the highly-swept and blunt leading edge portion of the configuration, although all surfaces of the model were analyzed and tripped for the experiment.

In this paper, we present the hybrid traditional/CFD-based approach that was developed in the course of the test planning to guide boundary-layer transition sizing and placement for the first test of the NASA/Boeing ERA HWB configuration at the NASA Langley 14- by 22-Foot Subsonic Tunnel. ${ }^{5}$ Measurements with infrared thermography confirmed the success of this approach, and the trips were sustained for subsequent testing of the model at the AEDC National Full-Scale Aerodynamics Complex (NFAC), 40- by 80-Foot Wind Tunnel ${ }^{6}$ (located at the NASA Ames Research Center). Details of the configuration, flow solvers and test conditions will be reviewed next followed by the transition strip analysis, development and confirmation.

\section{Configuration}

The $5.75 \%$ scale wind tunnel model of the ERA HWB in the LaRC 14- by 22-Foot Subsonic Tunnel is shown in Figure 3. This HWB wind tunnel model has the leading-edge slats deployed, vertical tails included, and flowthrough nacelles (FTN) mounted on the upper aft surface body. The model had a reference wing span of $12.228 \mathrm{ft}$. and a mean aerodynamic chord of $3.717 \mathrm{ft}$. The model is mounted on a sting strut that created the least interference with the model, as determined from an earlier computational study. ${ }^{7}$

Two configurations were used in the current study to determine effective and practical placement of forced boundary-layer transition trip dots on the experimental configuration; (i) a cruise wing configuration and (ii) a highlift wing configuration that had the leading-edge slat deployed in one position. Both configurations were modeled in 
the computational domain with the wind tunnel model sting, flow-through nacelles and pylons, and vertical tails. Neither configuration had the center elevon or other trailing-edge devices deflected.

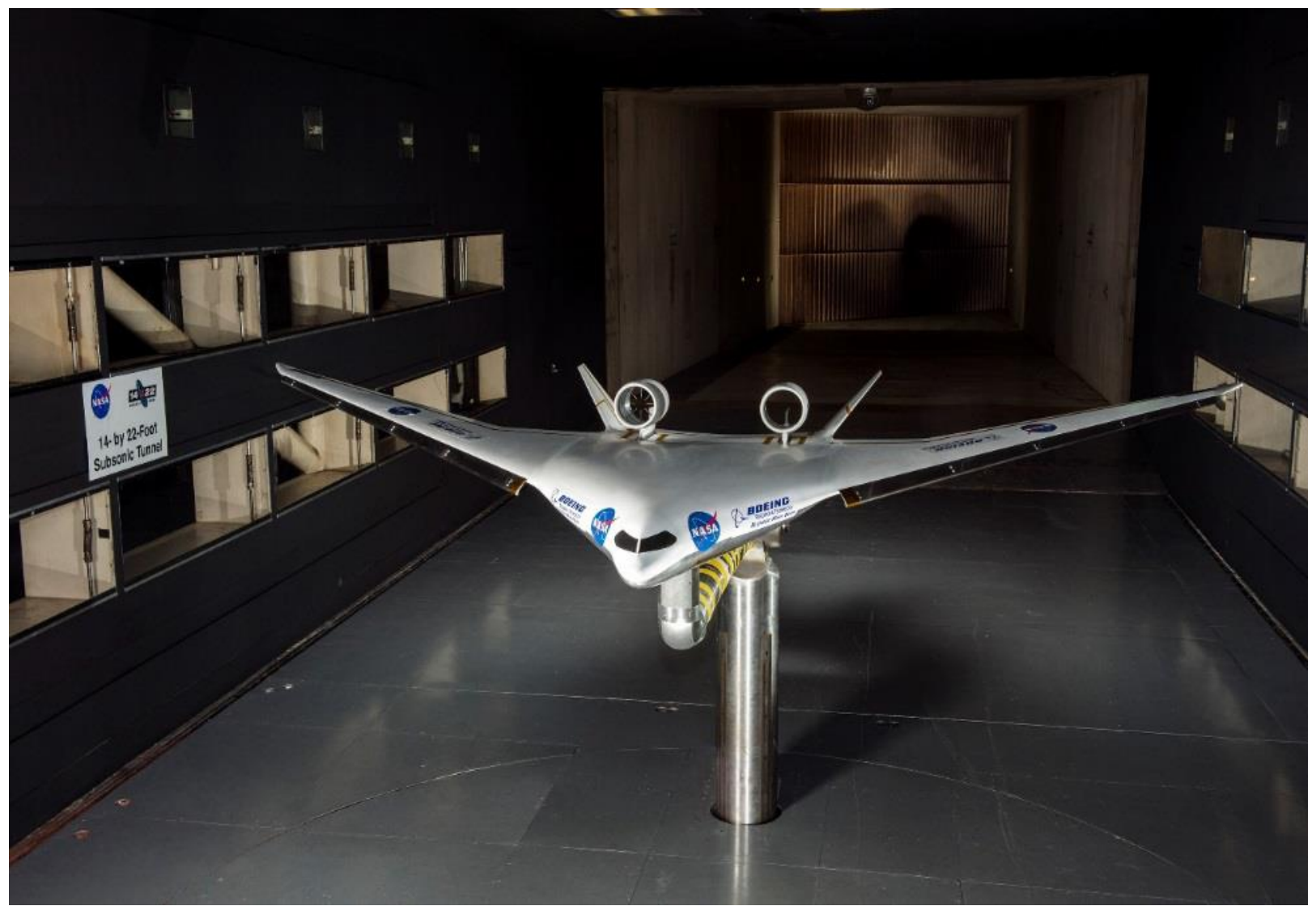

Figure 3. The 5.75\% ERA HWB wind tunnel model in the LaRC 14- by 22-Foot Subsonic Tunnel.

\section{Flow Solvers}

Two flow solvers were used for this analysis. The first was the unstructured grid code USM3D, ${ }^{1}$ and fully turbulent simulations were performed with USM3D using the Spalart-Allmaras turbulence model. ${ }^{2}$ The second was the overset-grid solver OVERFLOW, ${ }^{8}$ and transitional flow simulations were performed with OVERFLOW using the Langtry-Menter transitional flow model. ${ }^{9}$ Grids were generated following established practices for each method, and convergence was achieved for the methods also falling within the method established practice experiences. Prior to the current study, both USM3D/SA and OVERFLOW/SA had been used extensively in the ERA HWB project, and the codes had correlated well with each other in many applications. ${ }^{10}$

\section{A. USM3D computations}

The NASA Tetrahedral Unstructured Software System (TetrUSS) was used for this computational analysis. ${ }^{11}$ TetrUSS includes a model preparation tool (GridTool), grid generation software (VGRID, POSTGRID) and a computational flow solver (USM3D). The TetrUSS flow solver, USM3D, is a tetrahedral cell-centered, finite volume RANS method. The USM3D code has a variety of options for solving the flow equations and several turbulence models for closure of the RANS equations. ${ }^{11,12}$ The USM3D flow solver has internal software to calculate forces and moments. Additionally, the LaRC-developed code USMC6 was used for analyzing the solutions. ${ }^{13}$

All of the USM3D computations were performed at the Langley Research Center. The standard practices developed at NASA for USM3D were followed in all aspects of the computational study. This included use of the Spalart-Allmaras turbulence model ${ }^{2}$ for all USM3D results of this study. 


\section{Grid Generation}

The lofted outer mold line definition was exported from the CATIA solid model to an IGES file for input to GridTool ${ }^{14}$ for geometry preparation. Surface patches were created on the configuration and sources (that regulate the mesh resolution) were placed throughout the domain to accurately capture configuration characteristics. The output from GridTool was used to automatically generate the computational domain with the VGRID unstructured grid generation software. The VGRID software used an Advancing Layers Method to generate thin layers of unstructured tetrahedral cells in the viscous boundary layer, and an Advancing Front Method to populate the volume mesh in an orderly fashion. ${ }^{15,16}$

Finally, the POSTGRID software was used to close the grid by filling in any gaps that remained from the VGRID process. POSTGRID is automated to carefully remove a few cells surrounding any gaps in the grid and to precisely fill the cavity with the required tetrahedral cells (without gaps) to finalize the mesh.

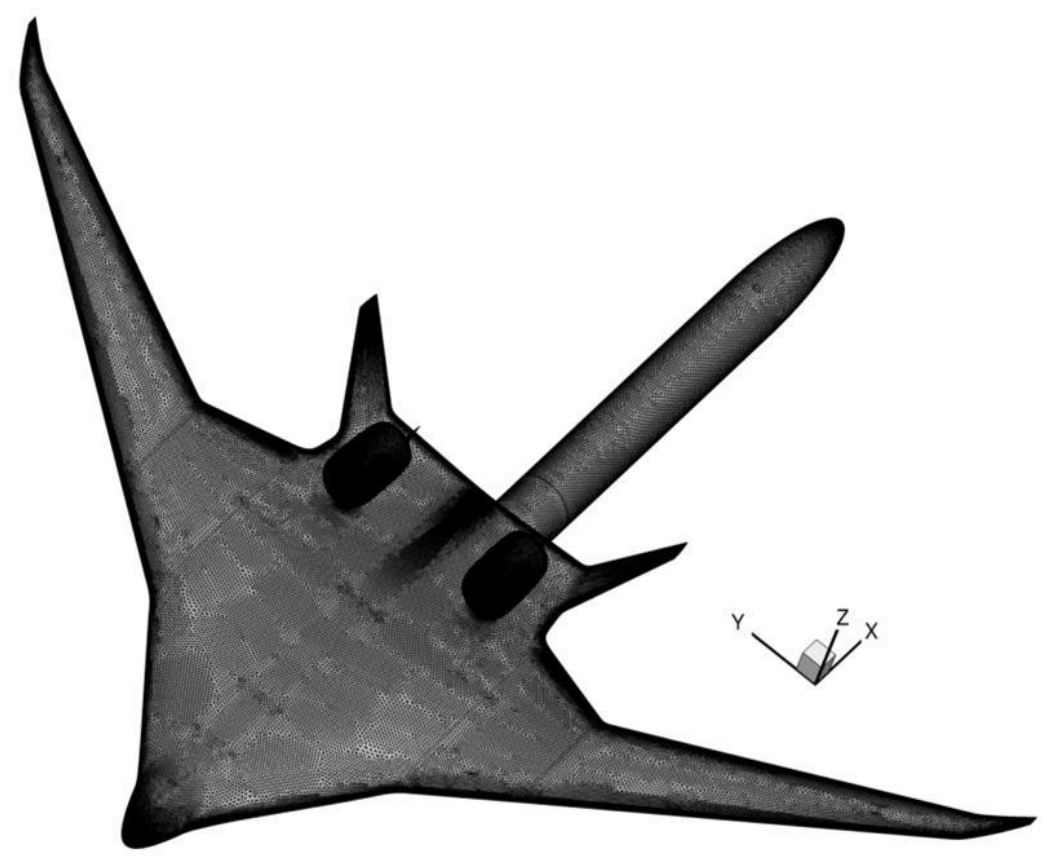

Figure 4. USM3D surface mesh.

The geometry and computational domain were specified in model-scale inches. The computational domain extended approximately 33 mean aerodynamic chord lengths from the configuration in the $\mathrm{x}, \mathrm{y}$ and $\mathrm{z}$ directions. The unstructured mesh contained 80 million cells for the full-span cruise wing configuration and 117 million cells for the full-span slat-deployed configuration. The first cell height in the boundary layer mesh was specified for $\mathrm{y}^{+}=0.5$. The upper surface mesh on the clean configuration with wind tunnel sting is shown in Figure 4.

\section{Computational Flow Solver and Solution Procedure}

This study used the implicit Gauss-Seidel scheme and the Roe flux difference-splitting scheme. The code was run in first-order spatial accuracy until the residual dropped two orders of magnitude. Then, the code automatically switched to generate second-order spatially accurate solutions. The SA turbulence model was used for all of the flow conditions. The SA turbulence model was implemented with a first-order advection term.

No-slip boundary conditions were used on all solid surfaces. A subsonic inflow boundary condition was used at the inflow face of the domain and an extrapolation boundary condition was used at the downstream outflow face of the domain. A characteristic inflow and outflow boundary condition was used along the far field, lateral faces of the domain.

The USM3D code computed the forces and moments at each iteration for the total configuration and for individual components that were specified. These values were used to track the convergence throughout the solution development. The Langley-developed post-processing tool, USMC6, was also used to extract data for postprocessing analysis.

\section{Convergence Criteria}

Two main criteria were used to monitor and determine USM3D solution convergence. First, a drop in residual ( $\mathrm{L}_{2}$-norm of the mean flow residuals) of at least two orders of magnitude was required. Second, the convergence of $C_{L}, C_{D}$ and $C_{m}$ was considered achieved when the maximum variation of the coefficient (Ra) over 2000 iterations was less than $0.1 \%$ of the average coefficient value (Av), also computed over 2000 iterations. These criteria, in conjunction with the above-mentioned grids and flow modeling, were sufficient to converge the HWB solutions.

Representative force and moment convergence and residual histories for USM3D are shown in Figure 5. The scales and averaged values of the force and moment coefficients have been removed from Figure 5, however, the level of detail in evaluating convergence remains. The maximum variation of the coefficient (Ra) over 2000 
iterations, normalized by the average coefficient (Av) in the same range, is shown as a percent in the upper right corner of the figure. The drop in solution and turbulence residuals is plotted as a function of iteration in the top left of the figure, and the overall drop in residuals is tabulated in the upper right side of the plot. This data for a representative solution had all coefficients well converged, with $\mathrm{Ra} / \mathrm{Av}=0.00 \%$ because the maximum variation of each coefficient over 2000 iterations was less than 0.00004 times the average coefficient value. Additionally, in this example, the solution and turbulence residuals dropped 6.7 and 5.7 orders of magnitude, respectively. All solutions were judged with this type of data in similar plots to Figure 5.
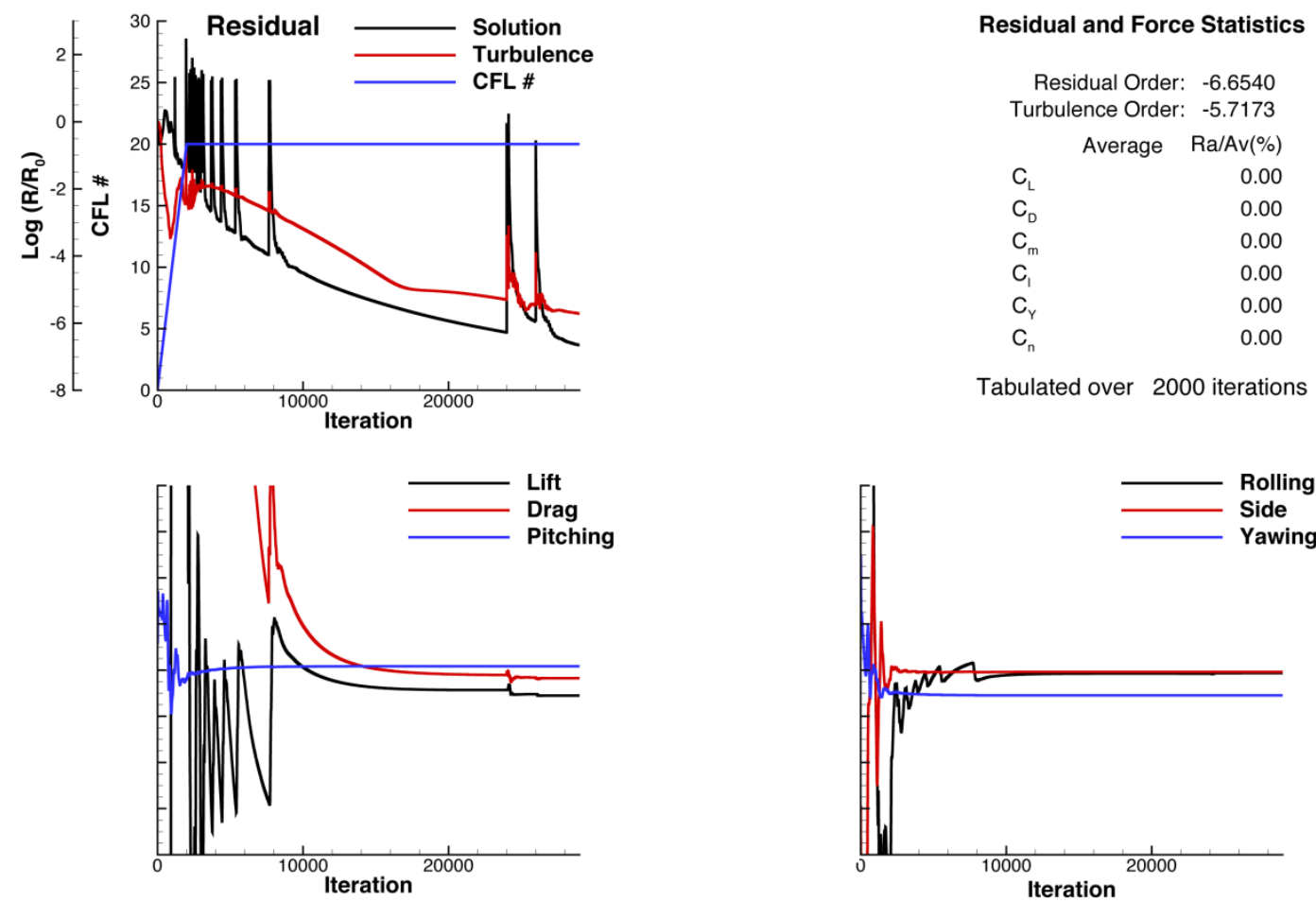

Figure 5. Representative force and moment convergence and residual histories for USM3D.

\section{B. OVERFLOW computations}

OVERFLOW simulations were performed using OVERFLOW ${ }^{8}$ version $2.2 \mathrm{~d}$ on a system of overset structured grids, with grid connectivity generated by Pegasus $5 .{ }^{17}$ The OVERFLOW solver for these solutions incorporated the Langtry-Menter turbulence model, a physical time step of 10, 5 inner (or sub) iterations, the HLLC upwind scheme, SSOR algorithm, van Albada limiter, time accurate mode, $\mathrm{CFL}_{\min }=10$, and $\mathrm{CFL}_{\max }=30$.

Guidance on placement and sizing of trip dots can be provided by CFD calculations using the Langtry-Menter transition model. The logic and details of this approach are described here. The information needed for this guidance comes from the laminar boundary layer development at otherwise flight-like condition, specifically, with flight-like surface streamlines, stagnation and separation lines, and pressure distribution. Neither a "fully turbulent" nor a fully laminar CFD result will suffice. The former fails because typical CFD transition (e.g., from the SST model) occurs very early, which contaminates the computed laminar boundary layer, while the latter will generally predict separated flow, which alters the gross flow feature like lift and stagnation lines. What is needed is a simulation that predicts transition to be slightly downstream of the true transition, but also predicts turbulent flow downstream and accurately models any flow separation that may occur.

The Langtry-Menter transition model can be used to obtain the needed data by running simulations with an artificially low level of free-stream turbulence. The transition location predicted by the Langtry-Menter model depends on an empirical correlation of transition's dependence on free-stream turbulence and $R_{\theta}$, an approximate easy-to-compute surrogate for $R e_{\theta}$, and history effects. If the free-stream turbulence in the CFD simulation is set to a lower value than in flight, the predicted transition should occur later than in flight. Langtry and Menter ${ }^{9}$ have demonstrated the dependence of transition on free-stream turbulence intensity. Furthermore, the Langtry-Menter model does not account for crossflow transition, which also contributes to predicting a delayed transition in many 
flows. This strategy can fail if the transition or overall flow characteristics are sufficiently different than flight behavior.

Langtry-Menter simulations were used to map the laminar boundary layer parameters, such as $R e_{\theta}, \theta, \delta^{*}$, and the shape factor $H_{12}$, over the relevant range of wind-tunnel test conditions. The pressure field is also generated by the simulations. The predicted transition location is easily recognized by a sharp drop in the shape factor. Suitable trip dot locations are where $R e_{\theta}$ is large enough, e.g., $R e_{\theta}>500$, followed by a suitable pressure gradient, neutral to adverse. The boundary layer thickness parameters $\theta$ and $\delta^{*}$, can be used to size the height of the trip dots.

\section{Grid Generation}

The OVERFLOW grids used in this study include the ERA 51a 0009D model and a representation of the LaRC 14- by 22-Foot Subsonic Tunnel. The ERA 51a model included the vehicle, Kruger flap, and flow thorough nacelles. The wind tunnel representation included a constant cross section of the test section, the model sting, and model support. The representation of the tunnel extended 1000 inches upstream and downstream of the model reference center. Pitch and sideslip were modeled by rotating the tunnel and support grids, while keeping the model and sting grids stationary. This is shown in Figure 6 for $\alpha=25^{\circ}$.

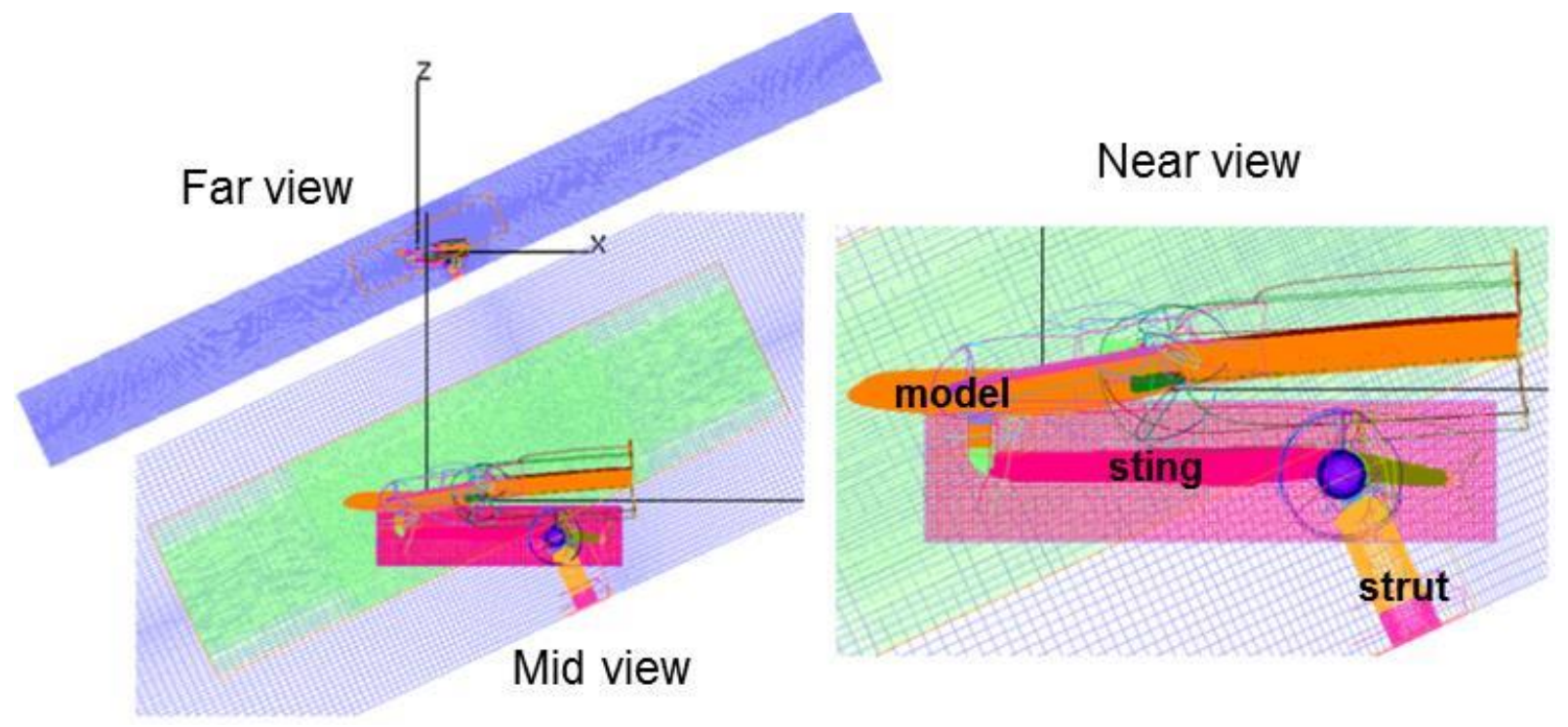

Figure 6. OVERFLOW grid.

Wake and sting box grids were included to better define the flow about the vehicle. All OVERFLOW grids were created using the Chimera Grid Tools for overset grids and conformed to best practices for structured grids. These practices included normal wall spacing that yield a $y^{+}$value less than 1 , wake box position and extent, and grid transition spacing. The hole-cutting process was completed using Pegasus $5 .{ }^{17}$ The total grid system included 82 grids for a total of slightly under 134 million grid points.

\section{Computational Flow Solver and Solution Procedure}

OVERFLOW simulations were performed using OVERFLOW ${ }^{8}$ version $2.2 \mathrm{~d}$ on a system of overset structured grids, with grid connectivity generated by Pegasus5. ${ }^{17}$ The Langtry-Menter turbulence model was used for all solutions. All solutions were started independently without a restart methodology from a previous solution. This was required because each of the grid system is unique, owing to the rotation of the tunnel grid system with respect to the model and sting. The OVERFLOW computation was executed to solution convergence.

Post processing of the OVERFLOW solutions for boundary layer properties was enabled through an analysis package OVF_MAN developed by one of the authors while at NASA Ames. The values of boundary layer parameters were computed from their formal definitions, by integrating normal to the vehicle surface until the local vorticity approached zero at the edge of the boundary layer. This integration is approximate in several respects: it integrates along the wall-normal grid coordinate (grid line), rather than a wall-normal vector, and only within individual grids that abut the vehicle surface. The edge of the boundary layer is taken to be the closest wall-normal position at which the local vorticity magnitude reaches a small fraction of the maximum vorticity; that fraction was typically 0.001 . Adverse effects of these approximations are weak in the leading-edge regions of interest. 


\section{Convergence Criteria}

All OVERFLOW solutions are run in time accurate mode until the convergence criteria were met. Convergence was determined using a fast Fourier transform (FFT) calculation in conjunction with the iterative forces and moments, $C_{L}, C_{D}$, and $C_{m}$. Solution residuals typically converge first, and the solutions are executed until forces and moment variations meet the convergence criteria: $C_{L}, \pm .004, C_{D}, \pm 0.01$, and $C_{m}, \pm 0.002$. An example of the convergence plots for the residuals as well as the lift, drag, and pitching moment coefficients is shown in Figure 7. Although the force and moment scales have been removed, the aforementioned criteria have been met in this example, and the residuals (shown for many components of the complete configuration) have dropped approximately 6 to 10 orders of magnitude.
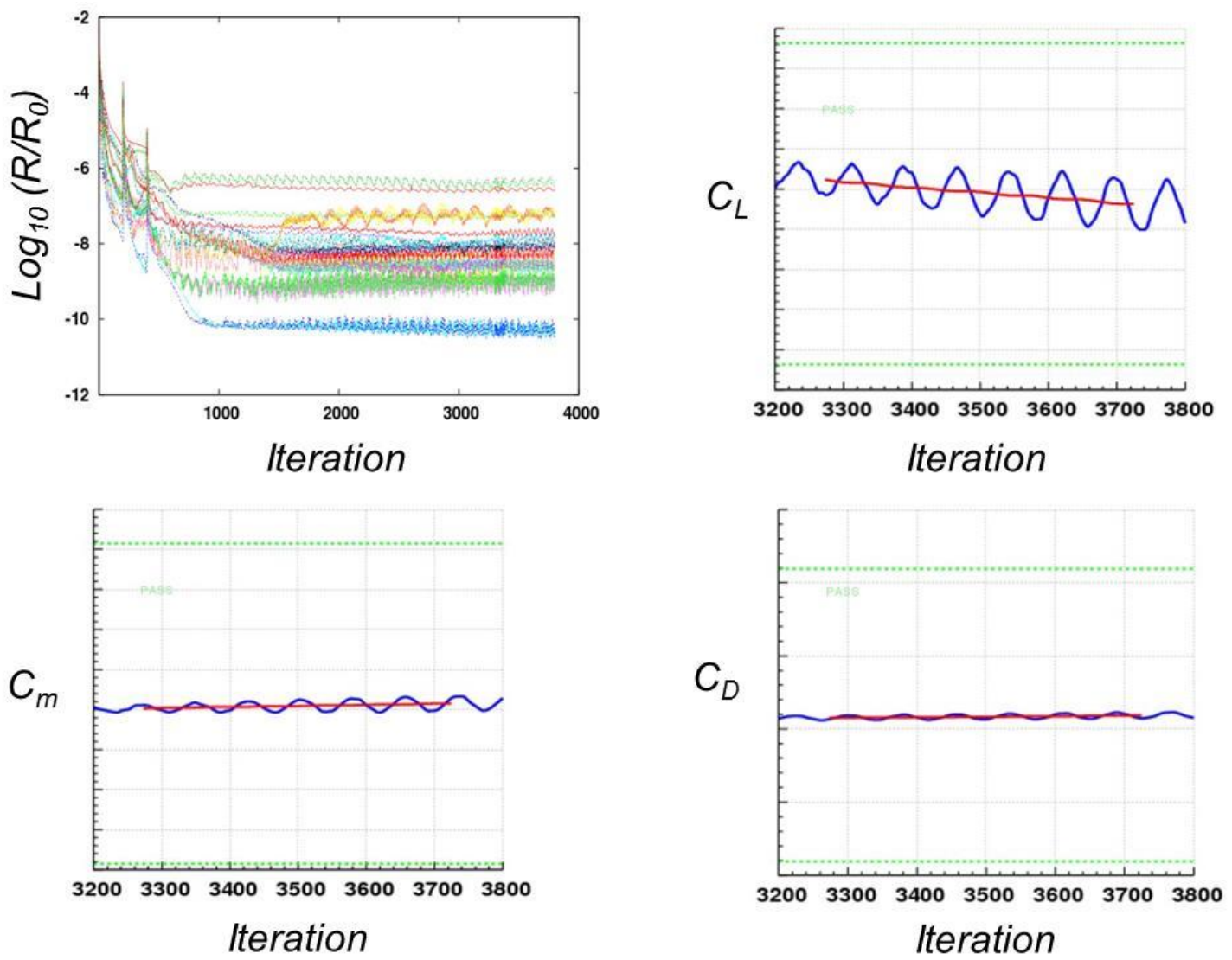

Figure 7. OVERFLOW convergence.

\section{Flow Conditions}

Flow conditions for the forced transition analysis matched planned wind tunnel testing conditions for the 5.75\% HWB model in the LaRC 14- by 22-Foot Subsonic Tunnel. Plans called for a free-stream dynamic pressure $q_{\infty}=60 \mathrm{psf}$, which corresponed to $M=0.20$ and $R e_{\text {cref }}=5.27 \times 10^{6}$. The analysis was organized into two angle-ofattack ranges. The first range corresponded to low-to-moderate angles of attack for the clean-wing configuration, $\alpha_{1}<\alpha<\alpha_{2}$. In most of this range, the wing was expected to sustain attached flow. The second range corresponded to moderate-to-high angles of attack for the configuration with the leading-edge slat deployed, $\alpha_{2}<\alpha<\alpha_{3}$. It was anticipated that forced transition strips might need to be altered between the first and second angle-of-attack ranges, and the break between the two ranges $\left(\alpha=\alpha_{2}\right)$ corresponded to the angle of attack in the test program for a model change to install the leading-edge slat. In this way, any changes to the forced transition strips would coincide with a scheduled model change, and thus only have a small impact on tunnel occupancy. 


\section{Transition Strip Analysis - Traditional Methods}

Traditional methods (e.g., Braslow et al. ${ }^{3}$ ) provided a foundation for the present work and the elements from that test technique that were used in the present analysis are now reviewed. Braslow et al. ${ }^{3}$ established a test technique for determining the location and size of trip particles to force a rapid transition of a boundary layer from laminar to turbulent flow, and the technique was demonstrated for applications to subsonic and supersonic flows. Both the location and size of the trip particles were established within bounds, so that effective tripping would result so long as the location and size of the trip element fell within those bounds.

As one example, a boundary-layer sketch is provided in Figure 8 that shows the placement of a roughness particle intended to force transition of a two-dimensional flat-plate boundary-layer flow. Local flow physics govern this physical process, and the forced-transition process is characterized with two local Reynolds numbers. The first is the length Reynolds number that is based on free-stream velocity and a distance ' $x$ ' from the origin of the boundary layer to the particle, $R e_{x}$. The second is a height Reynolds number that is based on roughness particle height ' $k$ ' and the local flow properties at the top of the roughness particle, $R e_{k}$. With this test technique, a minimum length Reynolds number was established for which tripping would occur. Tripping would also have to occur prior to natural transition, and hence the bounds on the length Reynolds number for effective boundary-layer tripping were $0.1 \times 10^{6}<R e_{x}<0.3 \times 10^{6}$. A

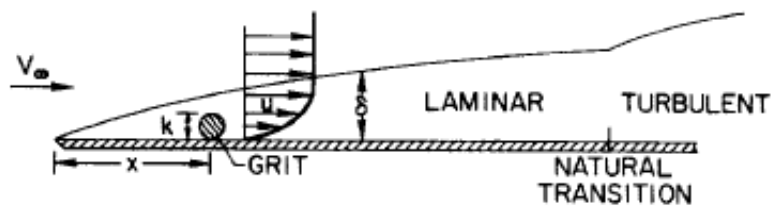

Figure 8. Boundary-layer roughness particle. From Braslow et al. ${ }^{3}$ minimum height Reynolds number was also established with this test technique for which tripping would occur. Trip particles would have to reside in the boundary layer, and hence the bounds on the trip particle size for effective boundary-layer tripping were $R e_{k}>600$ and $k / \delta<1$. With this test technique the laminar flow would be forced to transition to turbulent flow, and the transition would occur quickly, so long as the particles fell within these location and size bounds. The exact trip location and size had little influence on the preceding laminar flow since boundary layers are parabolic.

In practice, these guidelines tend to result in trip placement close to the leading edge for subsonic flows, and Braslow et al. ${ }^{3}$ pointed out that the favorable pressure gradient near the leading edge of an airfoil can extend the range for $R e_{x}$ to approximately $0.5 \times 10^{6}$. From the subsonic Braslow et al. ${ }^{3}$ methodology, the trip strip location for the testing of this HWB model would fall in a range $0.019<s / c_{r e f}<0.095$ measured along the surface using the airfoil guideline. Trip dot heights would be bounded by $0.48<k / \delta<1$ for the upstream $s / c_{\text {ref }}$ bound and $0.31<k / \delta<1$ for the downstream $s / c_{\text {ref }}$ bound.

Other flow metrics can result in modified trip locations. For supercritical wing applications, shock/boundarylayer interactions are important and Blackwell ${ }^{4}$ demonstrated that the forward trip locations produced thicker boundary layers than desired at the location of the upper surface transonic shock. He modified this technique by exploiting the relatively neutral-gradient transonic pressure distribution to establish an aft trip location to better simulate the boundary layer thickness at the wing shock location for ground-based testing. Aft trips have become another standard test technique for ground-based testing of supercritical wings.

For the current work, we retained the physics-based reasoning from the Braslow et al. ${ }^{3}$ work, but used CFD to define the environment to apply that reasoning. Basic trip dot sizing guidelines were retained, but the particular boundary layers and pressure gradients came from CFD analysis of the ERA HWB configuration. Details for this are described in the following section.

\section{Transition Strip Analysis - Hybrid Traditional/CFD-Based Method}

The most desired simulation for the HWB would have fully turbulent flow on the vehicle except for small regions near the leading edges. Here the flow would be laminar up to the location of forced transition, and of course this forced transition location is not known a priori. Transition could also require simulation along the lower surface attachment line. Neither code had benchmarked capability of simulating this flow. The CFD flow analysis for the current work was broken down into two steps. The first step was to determine transition strip locations, and this step followed some traditional methodology reasoning based on pressure gradients. Fully turbulent simulations were used for this step. The second step was to determine transition trip dot sizes at these locations, and this step followed other traditional methodology reasoning based on local boundary-layer properties. Simulations with free transition were used for this step. All analysis was performed at planned wind tunnel test conditions. 
Analyses were performed over angles of attack ranging from low-to-high values, $\alpha_{1}<\alpha<\alpha_{3}$, that were being planned for the initial wind tunnel test. The angle-of-attack analysis was broken into a low-to-moderate range $\left(\alpha_{1}<\alpha<\alpha_{2}\right)$ and a moderate-to-high range $\left(\alpha_{2}<\alpha<\alpha_{3}\right)$ that corresponded with a planned model change for the high-lift configuration of the wing. No analysis of sideslip effects was included.

The analysis of the configuration was subdivided into four spanwise regions that coincided with breaks in the leading-edge sweep, Figure 9. Pressure distributions and boundarylayer properties will differ between the less conventional thick and swept body-like portion of the configuration (inboard of sweep break 2) and the more conventional wing. It was also anticipated that the span stations for the sweep breaks could facilitate the practical implementation of segmented forcedtransition strips.

The emphasis for this work was on the highly-swept and blunt body portion of the HWB configuration (i.e., inboard of sweep break 2), and the hybrid traditional/CFD-based approach will be presented for this

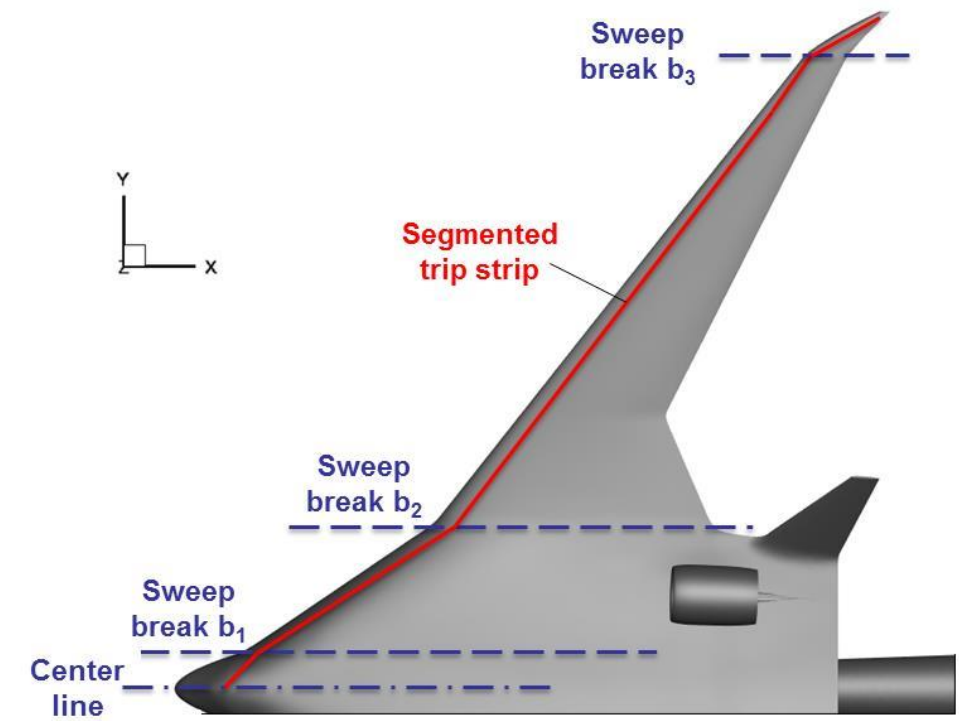

Figure 9. Leading-edge sweep breaks for subdivision of boundary layer trip strip analysis. portion first for the two angle-of-attack ranges. Discussion for the wing and other components will follow.

\section{A. Body, low-to-moderate angles of attack}

\section{Initial estimate for transition strip location}

As a first step in the current analysis, fully turbulent simulations were performed using USM3D with the SpalartAllmaras turbulence model. This use of CFD was to account for pressure gradient effects near the leading edge. The focus for this step was to identify regions of minimum pressure coefficient near the leading edges so that trip strips could be placed slightly ahead of this location (i.e., before the advent of adverse pressure gradients) as an initial estimate. The analysis was performed for the range of angles of attack of practical interest to the test program. Some typical results are shown in Figure 10 and Figure 11 for $\alpha_{1}<\alpha<\alpha_{2}$.

In Figure 10, angle-of-attack effects are shown near the leading edge at a span station between sweep breaks 1 and 2. Here it is seen that the location of the uppersurface suction peak, and subsequent onset of the adverse pressure gradient, varied linearly with angle of attack and, although the scales have been removed, a

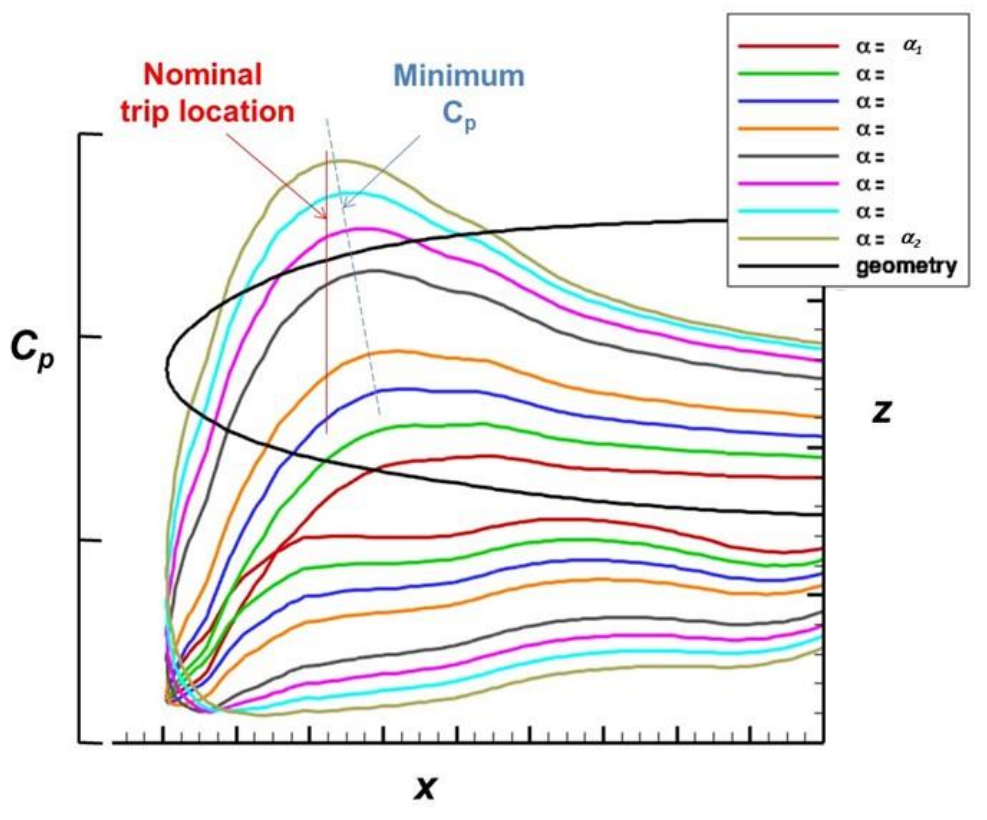

Figure 10. Angle-of-attack effects on surface pressure coefficients close to leading edge. $M=0.2, R e_{\text {cref }}=5.27 \times 10^{6}, \alpha_{1}<\alpha<\alpha_{2}, b_{1}<y_{a}<b_{2}$. key observation was that the minimum $C_{p}$ location only had a modest variation with angle of attack. This indicated 
that a single trip location could be possible for much of the angle-of-attack range shown. Similar results were obtained at other span stations.

In Figure 11, upper-surface pressure contours are shown for the highly-swept portion of the configuration at one angle of attack, $\alpha=\alpha_{a}$. Here the key observation was that the trough of minimum pressures forms a roughly straight line aft of the leading edge. This indicated that a single straight-line transition strip could be positioned ahead of this trough to cover this portion of the model. Similar results were obtained at other angles of attack, and a piecewise linear nominal trip location extending to the configuration centerline is shown by the dashed red line in the figure. There was very little change in the sweep of the trip location at sweep break station 1 , $y=b_{1}$. Figure 11 also includes surface streamlines, and the nominal trip location from the pressure analysis also exhibited

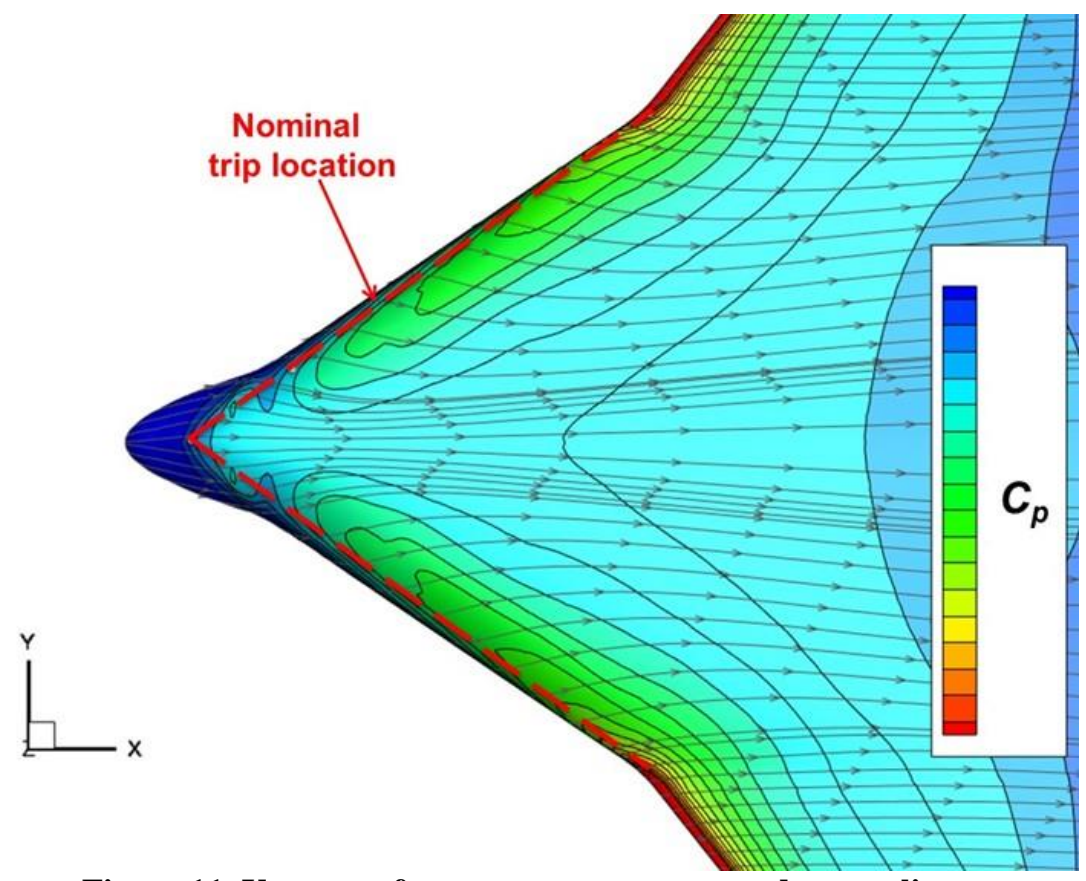

Figure 11. Upper surface pressure contours and streamlines.

$$
M=0.2, \boldsymbol{R e}_{\text {cref }}=5.27 \times 10^{6}, \alpha_{1}<\alpha_{a}<\alpha_{2} .
$$

effective streamline crossing for boundary-layer tripping. These results, and related analysis for other angles of attack, indicated that one strip could be positioned on the upper surface to potentially trip the boundary layer for this inner portion of the HWB configuration for the low-tomoderate angle-of-attack range analyzed. These positions were slightly further aft of positions that would have resulted from Braslow et al. ${ }^{3}$

The same pressure-gradient and streamline analysis was applied to the lower surface, but with very different results due to the usual distinctions of a lower surface flow, such as: (i) the further aft location of the pressure minima, (ii) the milder longitudinal pressure gradients (see also Figure 3), and (iii) the streamline characteristics associated with lower surface attachment line. One example of the pressure and streamline field is shown in Figure $\mathbf{1 2}$ for an angle of attack $\alpha=\alpha_{b}$. The principles to guide transition strip placement on the lower

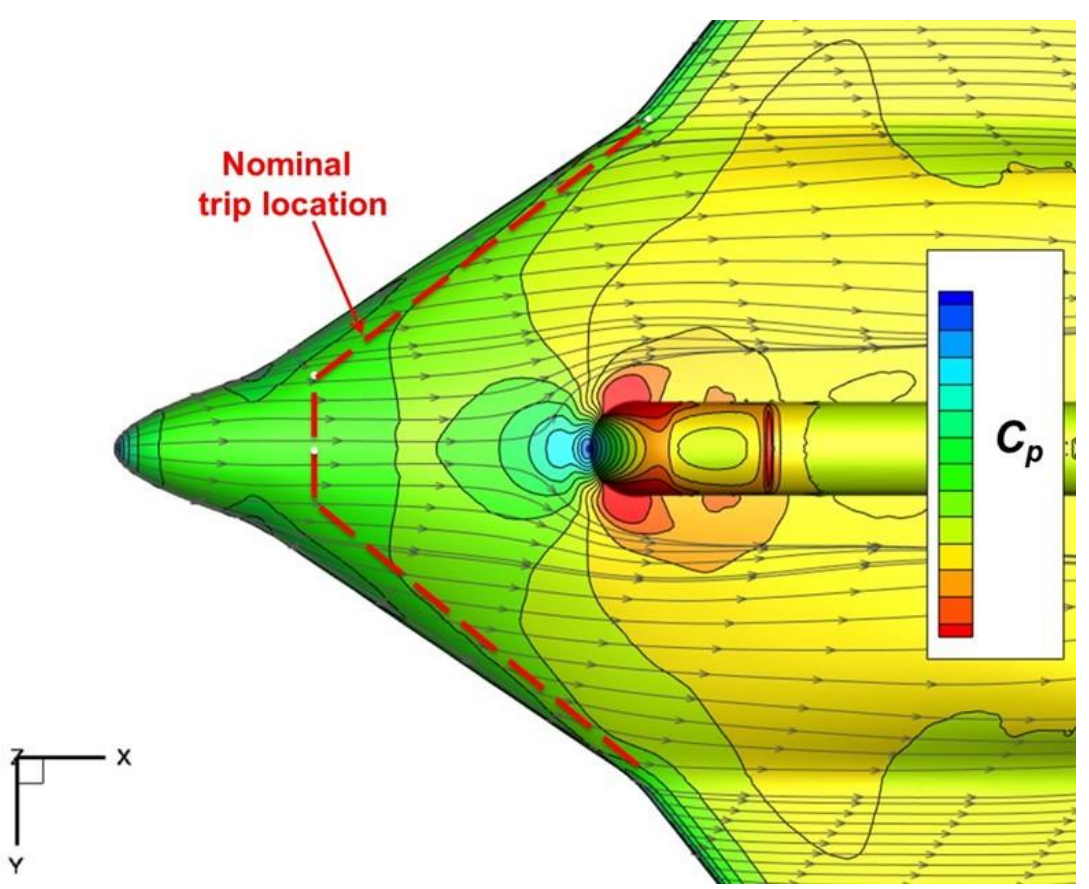

Figure 12. Lower surface pressure contours and streamlines.

$$
M=0.2, \boldsymbol{R e}_{\text {cref }}=5.27 \times 10^{6}, \alpha_{1}<\alpha_{b}<\alpha_{2} .
$$

surface were the same as for the upper surface, but the manifestation of the principles due to these local flow features resulted in a different, although still simple, nominal transition strip location. The nominal location was 
further aft on the lower surface than on the upper surface, but not as close to the minimum pressure coefficient on the lower surface. These positions were further aft of positions that would have resulted from Braslow et al. ${ }^{3}$

It was recognized that refinements to these trip strip locations could come from the subsequent analysis performed for trip-dot sizing with OVERFLOW and the transitional Langtry-Menter turbulence model.

\section{Transition dot sizing}

It must be recalled that the boundary layers to be tripped are, by the very nature of this task, laminar, and so laminar boundary layer properties are needed in the region of the trip strips for consideration of boundary-layer trip dot sizing. All the USM3D/SA results used to this point were fully turbulent. To obtain laminar boundary layer properties near the leading edges of the HWB, OVERFLOW was used in conjunction with the Langtry-Menter (LM) transitional turbulence model. The transition location predictions themselves were not anticipated to necessarily represent the HWB flow, but the thought was that the laminar solution properties near the leading edges could be useful for the present purposes. OVERFLOW also had a very useful boundary-layer analysis package (OVF_MAN) available to extract boundary-layer properties (e.g., displacement thickness, shape factor) that would be needed for this analysis.

Initial OVERFLOW/LM computations were performed at selected conditions to compare with the previous USM3D/SA results and to assure that there were no unanticipated discrepancies between the two formulations. It was anticipated that the pressure distributions near the leading edges should be similar between the two simulations for conditions that sustained attached flow, and the results of this assessment were very favorable. One example is shown in Figure 13 for pressure distributions near the leading edge at a span station between sweep breaks 1 and 2. The correlation between the two computations is very good. This correlation is representative of results from other span stations, and from other angle-of-attack conditions within this low-to-moderate angle-of-attack range, so long as the flow was attached.

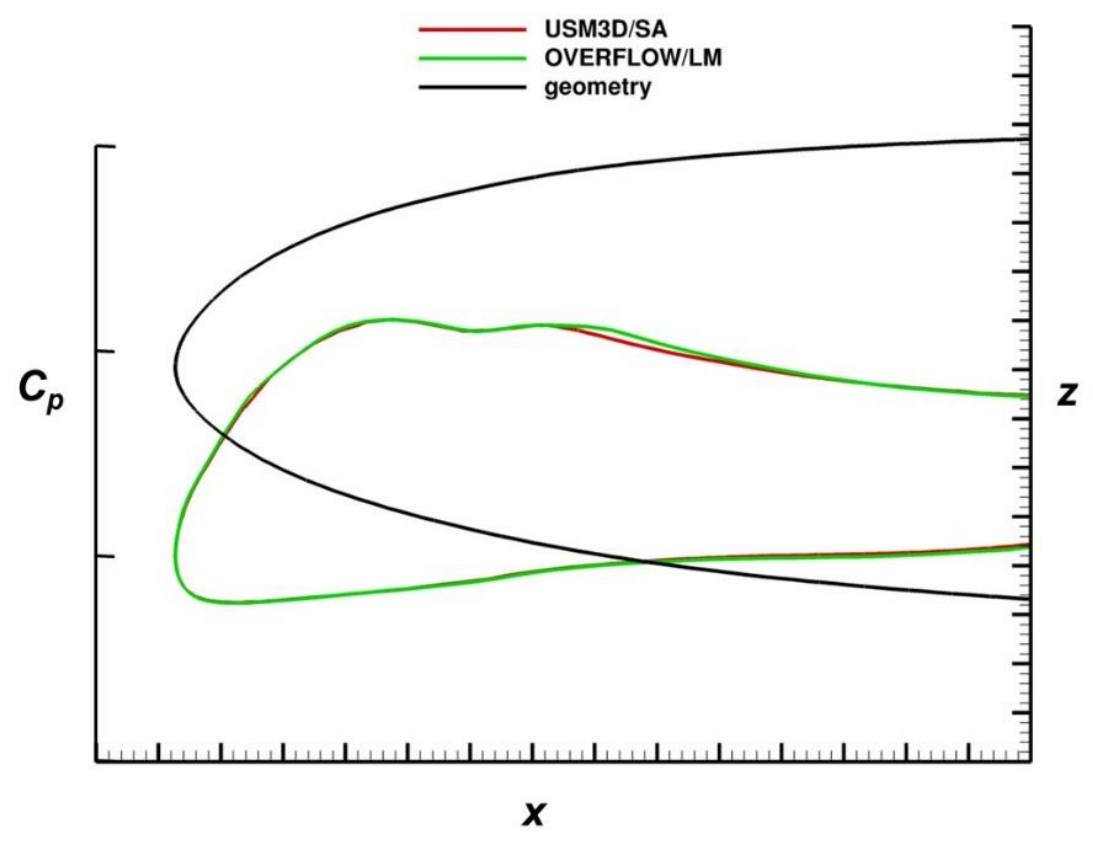

Figure 13. Pressure comparison, USM3D/SA and OVERFLOW/LM. Low-to-moderate angle-of-attack range. $M=0.2, \operatorname{Re}_{\text {cref }}=5.27 \times 10^{6}, \alpha_{1}<\alpha_{a}<\alpha_{2}, b_{1}<y_{b}<b_{2}$. 
The OVERFLOW/LM simulations, however, did produce transitional results with extensive regions of laminar flow. An example is shown in Figure 14 (upper surface) and Figure 15 (lower surface) in terms of the boundary layer shape factor $H_{12}=\delta^{*} / \theta$ at an angle of attack $\alpha=\alpha_{b}$. From boundary-layer theory, the twodimensional flow on a flat plate will produce $H_{12} \approx 2.59$ for laminar flow and $H_{12} \approx 1.27$ for turbulent flow; these values provide a useful guide for interpreting the results from transitional simulations. In Figures 14 and 15, laminar flow corresponds to the (hotter) red and orange colors; turbulent flow corresponds to the (cooler) green colors.

Figure 14 shows extensive regions of laminar flow near the leading edges of the body as well as on the wings, nacelles, and canted vertical tails. For the current application, the presence of laminar flow in the region of the leading edge is more important than the details of the transition front predicted

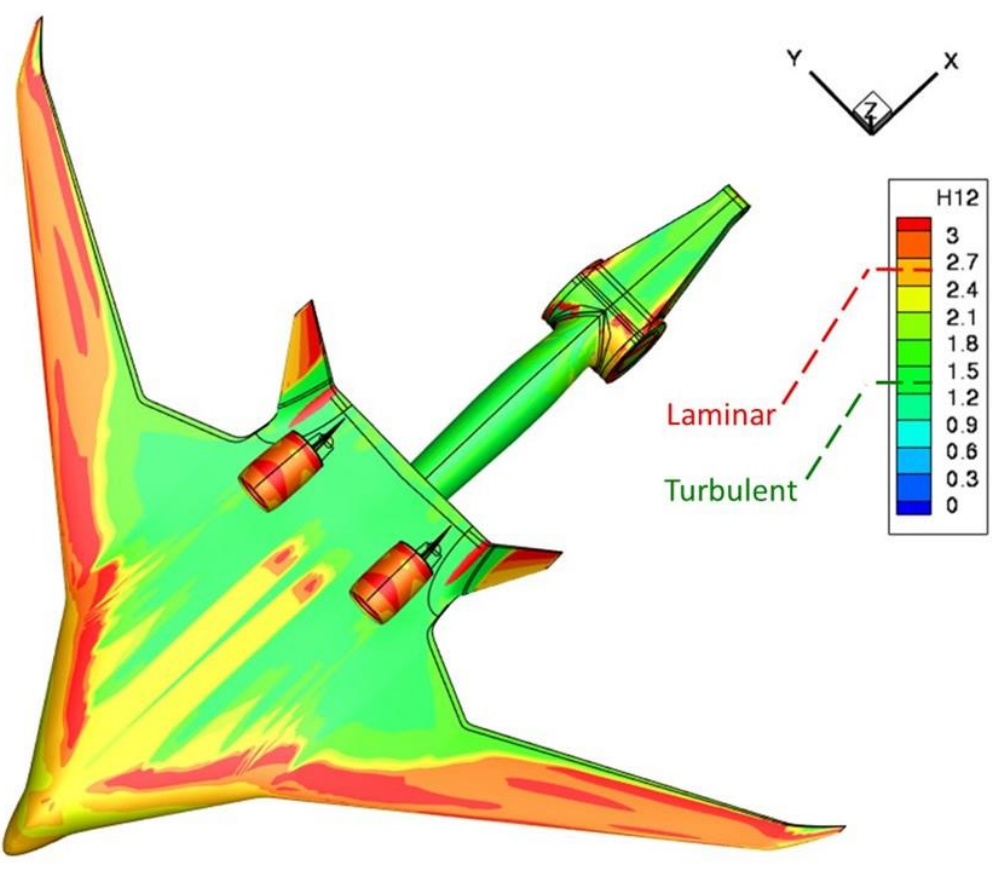

Figure 14. Upper surface boundary-layer shape factor, OVERFLOW/LM. $M=0.2, R_{c r e f}=5.27 \times 10^{6}, \alpha_{1}<\alpha_{b}<\alpha_{2}$. by the Langtry-Menter model.

The lower surface also had extensive regions of laminar flow predicted by the OVERFLOW/LM simulations. Figure 15 shows some of these results focused on the body portion of the configuration. Almost the entire forebody as well as the portion of the wing shown have laminar flow, and, thus, this simulation will support boundary layer analysis for trip-dot sizing in the regions identified from the USM3D/SA simulations.

Separation will occur where the wind tunnel support post enters the body on the lower surface, and the results from the transitional OVERFLOW/LM simulation in Figure 15 could imply that laminar and/or transitional flow physics are affecting this separation. Turbulent flow physics would be more desirable for this separation and, thus, model-support interference effects were noted as another reason for developing the particular forced-transition trip strips of the present study.

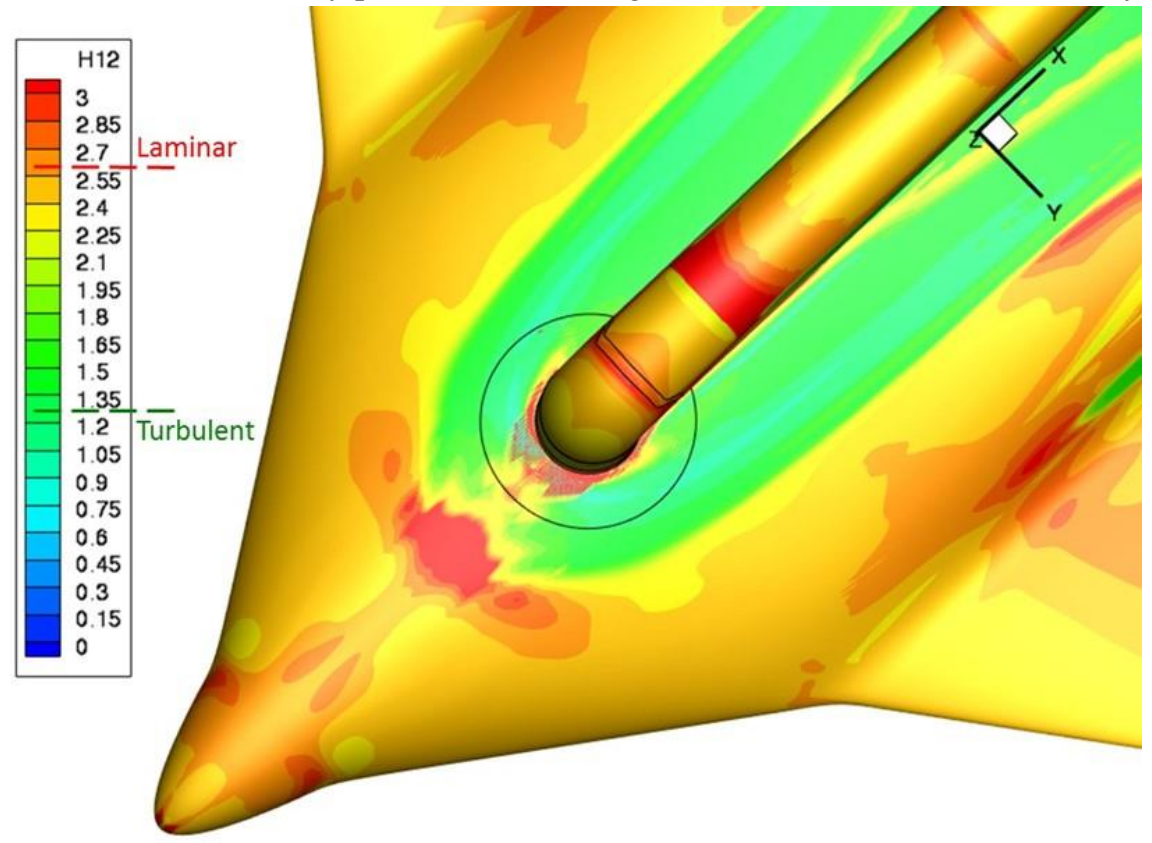

Figure 15. Lower surface boundary-layer shape factor, OVERFLOW/LM. $M=0.2, \operatorname{Re}_{\text {cref }}=5.27 \times 10^{6}, \alpha_{1}<\alpha_{b}<\alpha_{2}$. 
The OVERFLOW/LM boundary layer analysis included other conventional parameters such as boundary layer thickness, $\delta$, displacement thickness, $\delta^{*}$, and momentum thickness, $\theta$, but at the time this work was performed the momentum thickness Reynolds number, $R e_{\theta}$, was not readily available. It was decided to size the trip dots in terms of the Braslow et al. ${ }^{3}$ guidelines for the laminar boundary layers predicted from the OVERFLOW/LM simulations in the regions identified from the USM3D/SA analysis. The boundary layer displacement thickness, $\delta^{*}$, can be a more stable quantity to extract from numerical solutions, and the guidelines from Braslow et al. ${ }^{3}$ were implemented in terms of $\delta^{*}$ by using $\delta^{*} / \delta \approx 0.344$ from boundary layer theory for twodimensional laminar flow on a flat plate.

Contours of the boundary layer displacement thickness from the OVERFLOW/LM simulations are presented in

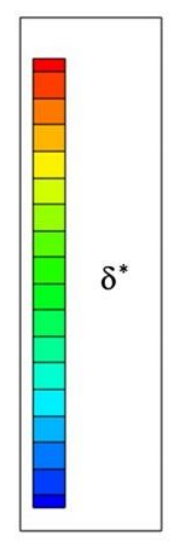

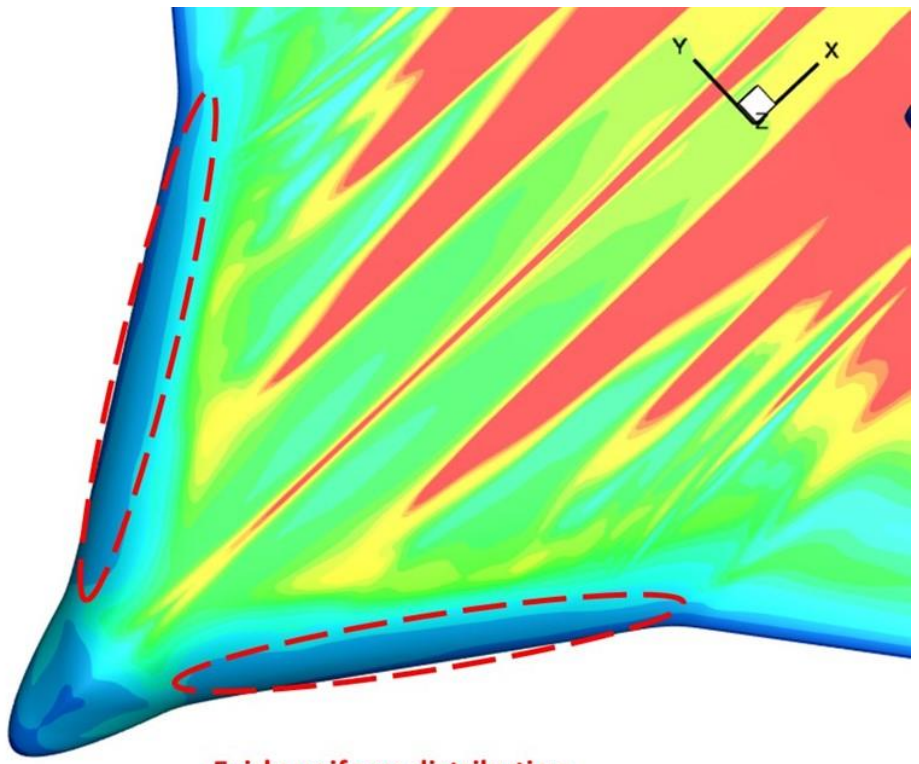

Fairly uniform distribution in vicinity of trip strips

Figure 16. Upper surface displacement thickness, OVERFLOW/LM. $M=0.2, R e_{c r e f}=5.27 \times 10^{6}, \alpha_{1}<\alpha_{b}<\alpha_{2}$.

Figure 16 for the upper surface and Figure 17 for the lower surface of the configuration at an angle of attack $\alpha=\alpha_{b}$ with a focus on the leadingedge region of the body. In both figures, the region of the proposed trip strips is indicated with a dashed ellipse. For both the upper and the lower surfaces, the variation in $\delta^{*}$ is relatively small within the regions for the proposed trip strips. This indicated that a single trip-dot size could be selected for the piece-wise linear trip strip locations identified from the USM3D/SA analysis. The trip-dot size would be different on the upper and lower surfaces. Figure 17 also indicates a second region on the lower surface, upstream of the transition strip geometry just discussed. This will be addressed subsequently.

With this information, a standard trip dot size was sought that was slightly larger than 1.16 times the displacement thickness; this corresponded to a dot height somewhat in excess of roughly 40 percent of the boundary layer thickness.

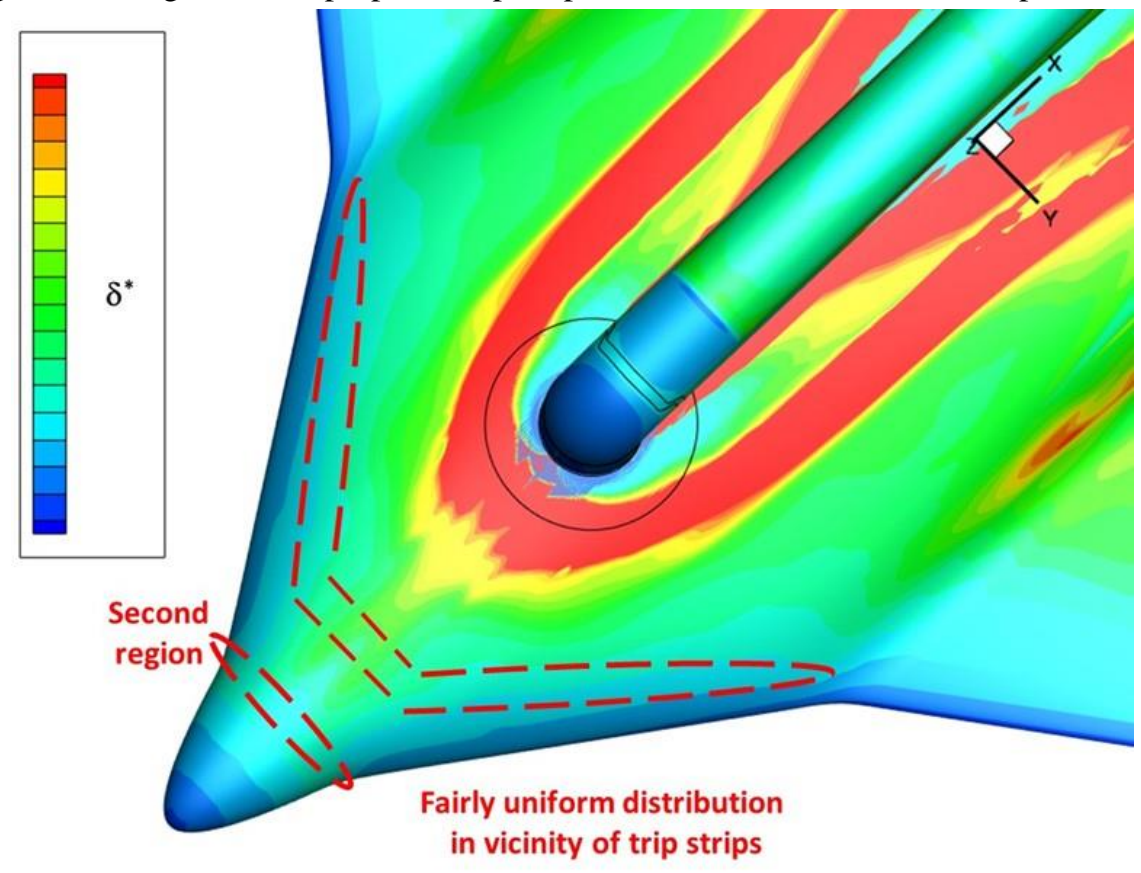

Figure 17. Lower surface displacement thickness, OVERFLOW/LM. $M=0.2, \boldsymbol{R e}_{\text {cref }}=5.27 \times 10^{6}, \alpha_{1}<\alpha_{b}<\alpha_{2}$. 
The OVERFLOW/LM simulations were performed for the angle-of-attack range under study and generally produced results similar to those discussed. The outcome from this analysis was that a single trip dot size could be used for the upper-surface strip and a single but different trip dot size used for the lower-surface strip on the body.

A medium-sized trip dot was selected for the upper-surface strip, and $0.4<k / \delta<0.8$ along the strip and over the low-to-moderate angle-of-attack range. The largest available trip dot was selected for the lower-surface strip, and $0.2<k / \delta<0.8$ along the strip and over the low-to-moderate angle-of-attack range. The low value of 0.20 was on the centerline, and fell outside of the basic guidelines from Braslow et al. ${ }^{3}$ for effective boundary layer tripping. A transverse nose ring with the same trip dot size was added upstream, where the boundary layers were thinner, to compensate for this result. The nose ring was positioned at the first sweep break station $\left(y=b_{1}\right)$ and is identified as the second region in Figure 17. No changes to the strips were indicated from the OVERFLOW/LM analysis for this low-to-moderate angle-of-attack range and, as a consequence, the application of the resultant trip dots was very practical.

\section{B. Body, moderate-to-high angles of attack}

The same analysis approach from the low-to-moderate angle-of-attack range was used for the moderate-to-high angle-of-attack range $\left(\alpha_{2}<\alpha<\alpha_{3}\right)$. For this angle-of-attack range, the HWB configuration included a baseline leading-edge slat that was rigged at one condition.

Comparisons between turbulent USM3D/SA and transitional OVERFLOW/LM simulations were again assessed for this moderate-to-high angle-of-attack range. Some larger differences were observed, particularly when separated flow was present for the higher angles of attack, but in general the comparisons near the leading edge between the two results were still very good. An example of this good $C_{p}$ comparison between the codes near the leading edge is shown in Figure 18 for an angle of attack $\alpha=\alpha_{c}$.

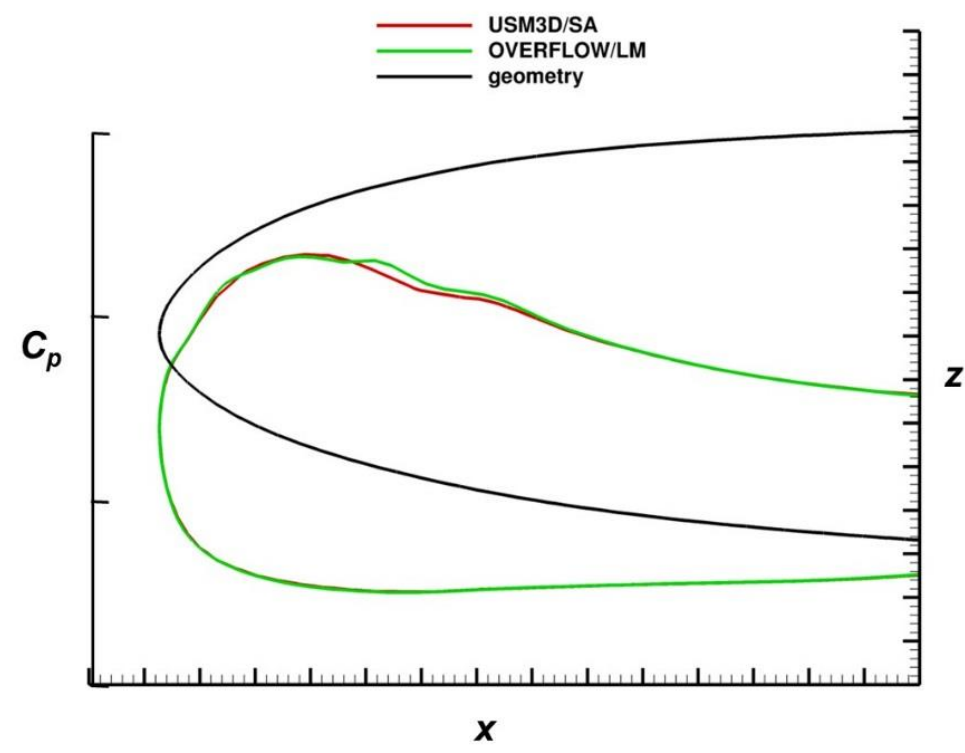

Figure 18. Pressure comparison, USM3D/SA and OVERFLOW/LM. Moderate-to-high angle-of-attack range. $M=0.2, \boldsymbol{R e}_{\text {cref }}=5.27 \times 10^{6}, \alpha_{2}<\alpha_{c}<\alpha_{3}, b_{1}<y_{b}<b_{2}$. 
Upper-surface pressure coefficients were assessed in the vicinity of the leading edge for variation with angle of attack and variations along the upper surface. Once again, the variation in peak pressure coefficient with angle of attack was small enough to propose a single trip location and near the leading edge, and an example of the spatial variation in surface pressure coefficient is shown in Figure 19 for an angle of attack $\alpha=\alpha_{c}$. The minimum pressure trough is still a uniform distance aft of the leading edge, and the same location from the low-tomoderate analysis could be used for this moderate-tohigh angle-of-attack range. This trip location also demonstrated acceptable streamline crossing.

At the moderate-to-high angle-of-attack conditions the lower surface pressure contours and streamline patterns differed significantly from the low-to-moderate angle-of-attack regime. An

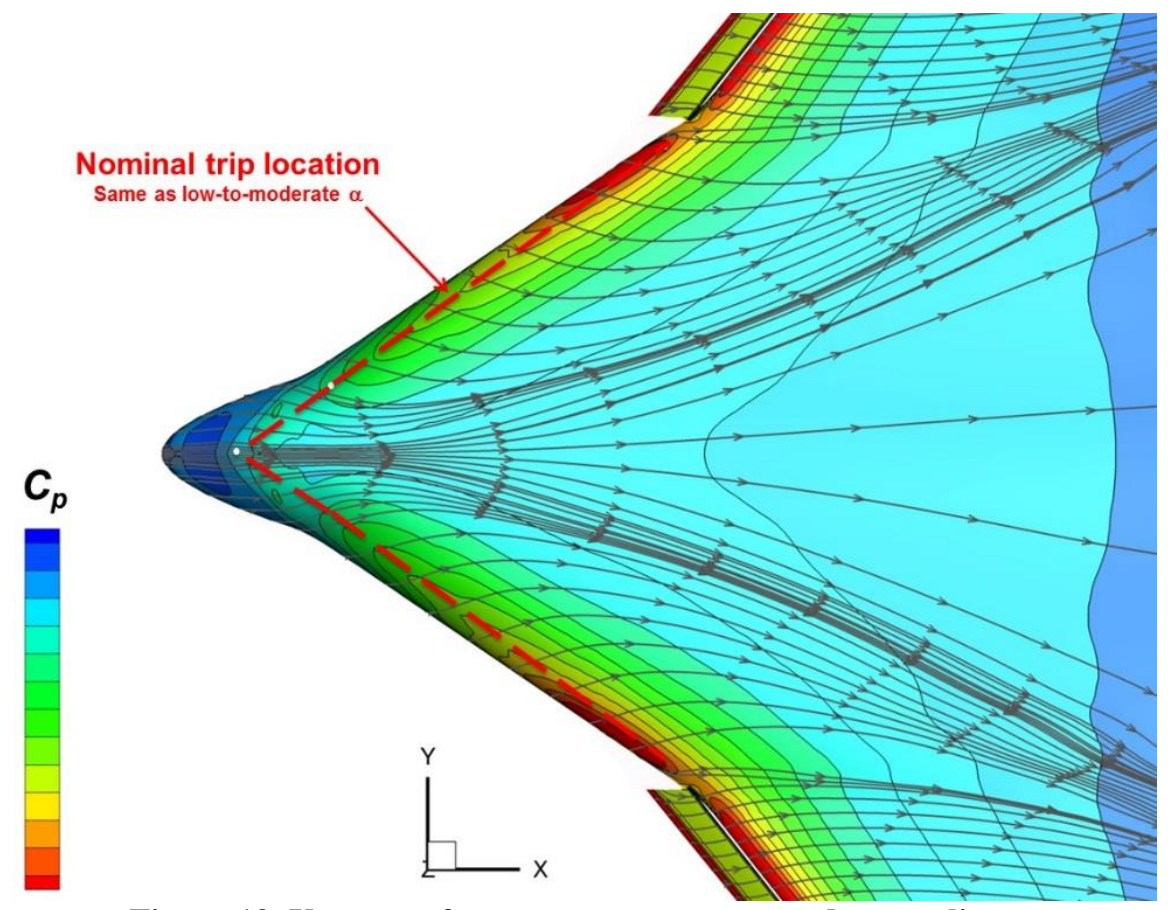

Figure 19. Upper surface pressure contours and streamlines.

$$
M=0.2, \boldsymbol{R e}_{\text {cref }}=5.27 \times 10^{6}, \alpha_{2}<\alpha_{c}<\alpha_{3} \text {. }
$$
example is shown in Figure 20 for an angle of attack $\alpha=\alpha_{c}$. The stagnation point and subsequent attachment line are swept and have shifted downstream such that the low-to-moderate trip location would no longer trip the lower surface flow. A simple chevron trip strip geometry was chosen that retained the trip strip anchor points at the centerline and the sweep break 2 stations. The nose ring trip strip was retained on the configuration from low angle-of-attack testing for this moderate-to-high angleof-attack testing.

Transitional flow analysis from OVERFLOW/LM still demonstrated laminar flow in the vicinity of the proposed trip strips, and sample results are presented in Figure 21 for the upper surface and Figure $\mathbf{2 2}$ for the lower surface at an angle of attack $\alpha=\alpha_{d}$. On the upper surface (Figure 21) the region of laminar flow is much smaller compared to the low-tomoderate angle-of-attack analysis, and this is likely due to the increased adverse

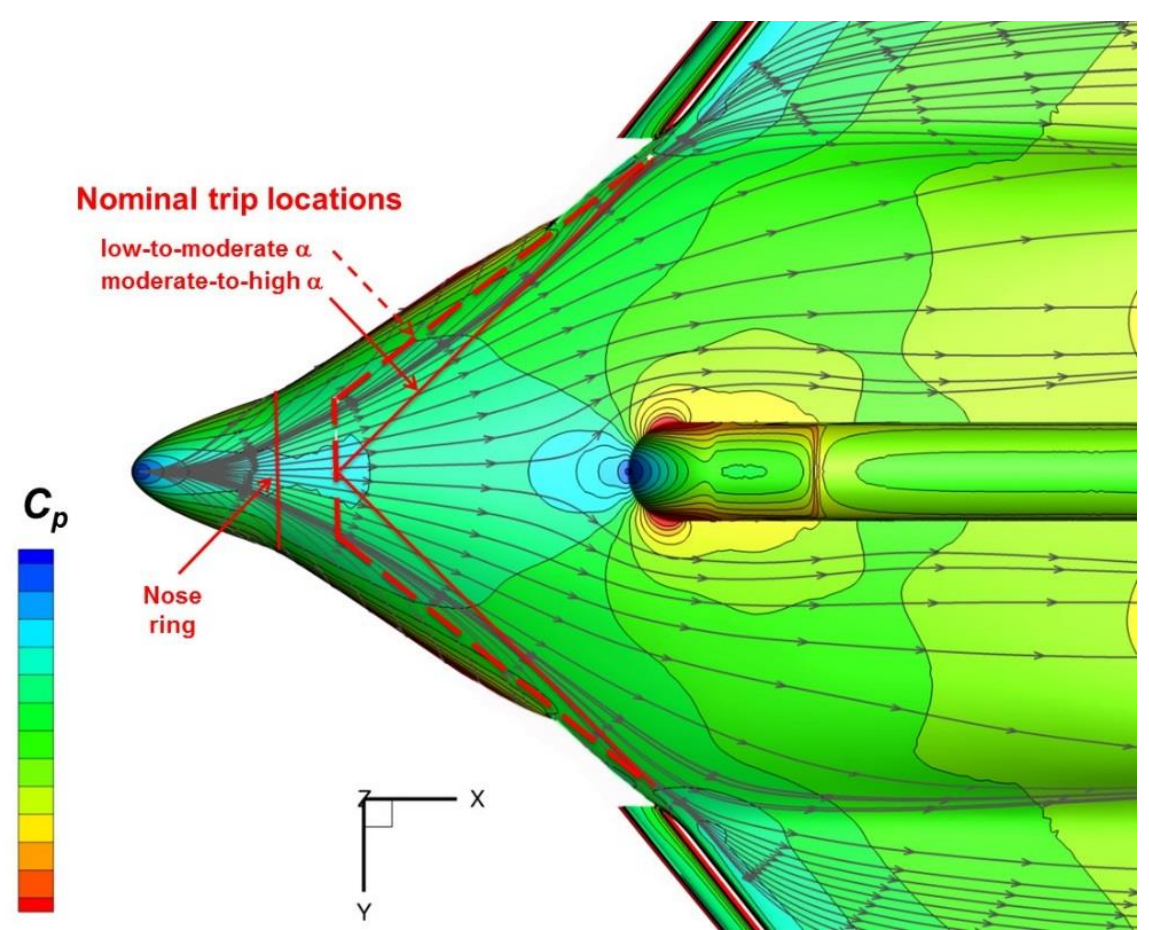

Figure 20. Lower surface pressure contours and streamlines. $M=0.2, \boldsymbol{R e}_{\text {cref }}=5.27 \times 10^{6}, \alpha_{2}<\alpha_{c}<\alpha_{3}$. 
pressure gradients near the leading edge in association with these higher angles of attack. The lower surface (Figure 22) still demonstrated a significant extent of laminar flow on the body.

The regions of laminar flow were again used to extract the boundary layer displacement thickness, $\delta^{*}$, to guide trip dot size selection. Results for both the upper surface (Figure 23) and the lower surface (Figure 24) demonstrated, once again, that the distributions of the displacement thickness were sufficiently uniform in the vicinity of the proposed trip strips, and a single trip dot size was chosen for each trip strip segment. These results are shown at a representative angle of attack $\alpha=\alpha_{d}$. The size resulted from a compromise over the angleof-attack range and location of the strip segment.

On the upper surface, the same trip dots from the lowto-moderate angle-of-attack

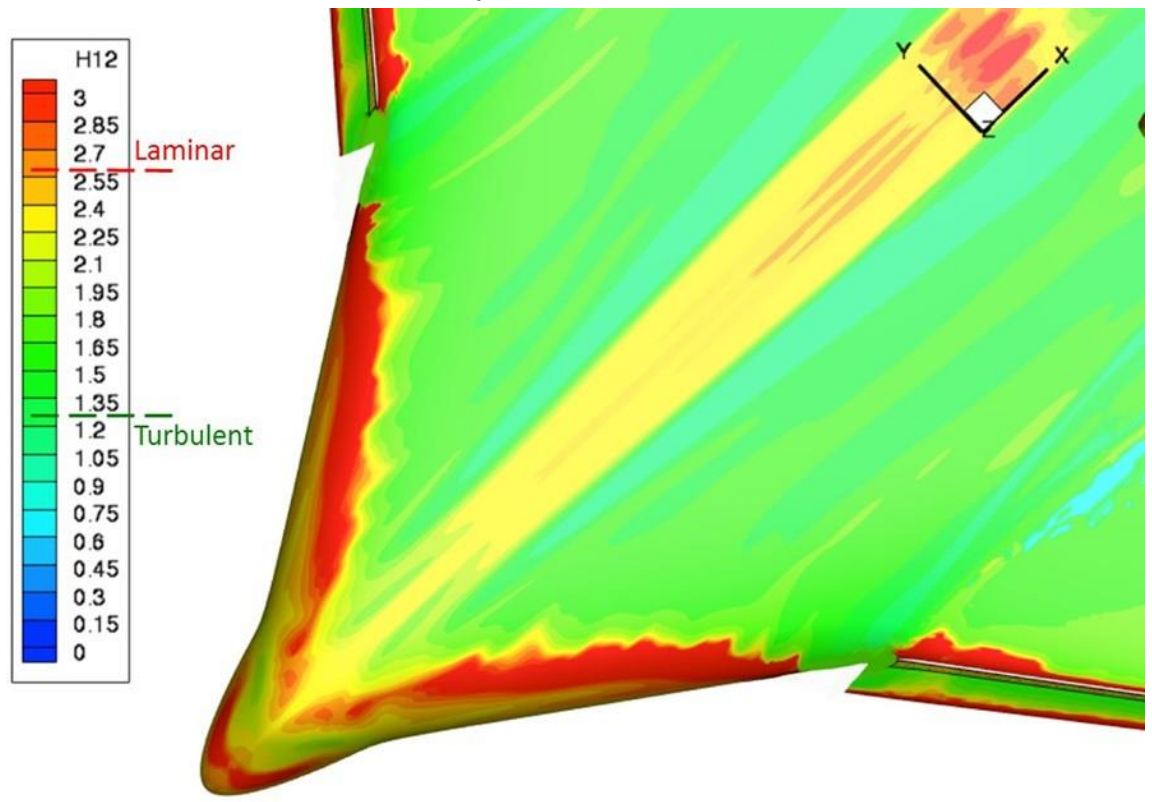

Figure 21. Upper surface boundary-layer shape factor, OVERFLOW/LM. $M=0.2, \boldsymbol{R e}_{\text {cref }}=5.27 \times 10^{6}, \alpha_{2}<\alpha_{d}<\alpha_{3}$. analysis were found to be acceptable at the moderate-to-high angle-of-attack conditions. In this application the dots resulted in $0.4<k / \delta<0.6$ along the strip and over the moderate-to-high angle-of-attack range.

The final trip strip location for the lower surface was sufficiently different for the moderate-to-high angle-ofattack testing conditions, as compared to the low-tomoderate angle-of-attack testing conditions, to warrant changing the patterns. However, this change corresponded with a major model change for installation of the leading-edge slat, and thus had a minimal impact on the tunnel occupancy time. The same size dots from the low-to-moderate angle-ofattack analysis were used and $0.3<k / \delta<0.5$ along the strip and over the moderate-to-high angle-of-attack range. The same transverse nose ring was retained for the moderate-tohigh angle-of-attack testing.

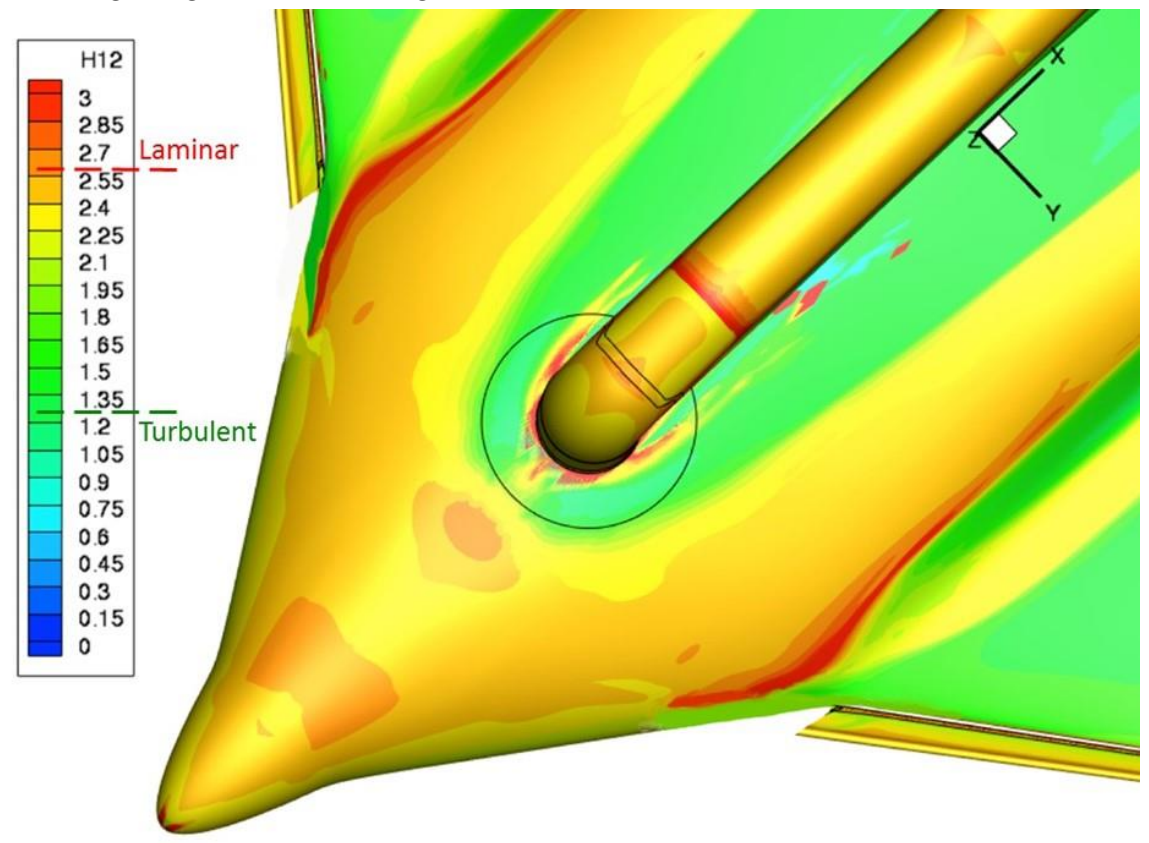

Figure 22. Lower surface boundary-layer shape factor, OVERFLOW/LM. $M=0.2, R_{\text {cref }}=5.27 \times 10^{6}, \alpha_{2}<\alpha_{d}<\alpha_{3}$. 


\section{Wing, Nacelle, and Canted Vertical Tails}

The same analysis was also performed for the wing, nacelles, and canted vertical tails. A brief summary of the outcomes from this analysis follow.

For low-to-moderate angles of attack, the clean wing exhibited leading-edge flow properties much as would be expected from other transport wing experiences. The present analysis resulted in small trip dots being placed close to the leading edges. The dot size was uniform and the trip strips were segmented straight lines between the leading-edge sweep break points. At high angles of attack, the wing had the leadingedge slat installed, and no trip dots were applied. The judgement was that the combination of the slat and its mounting brackets would be sufficient to trip the wing flow.

The nacelles and canted vertical tails also exhibited leading-edge flow properties at low-to-moderate angles of attack much as would be expected from prior transport experiences. Local flow properties for this HWB configuration were once again used with the same analysis approach described above to arrive at trip dot size and placement for these components. The dots were small and close to the leading edges. Analysis for the moderate-to-high angle of attack range indicated that the same tripping arrangement could be used.

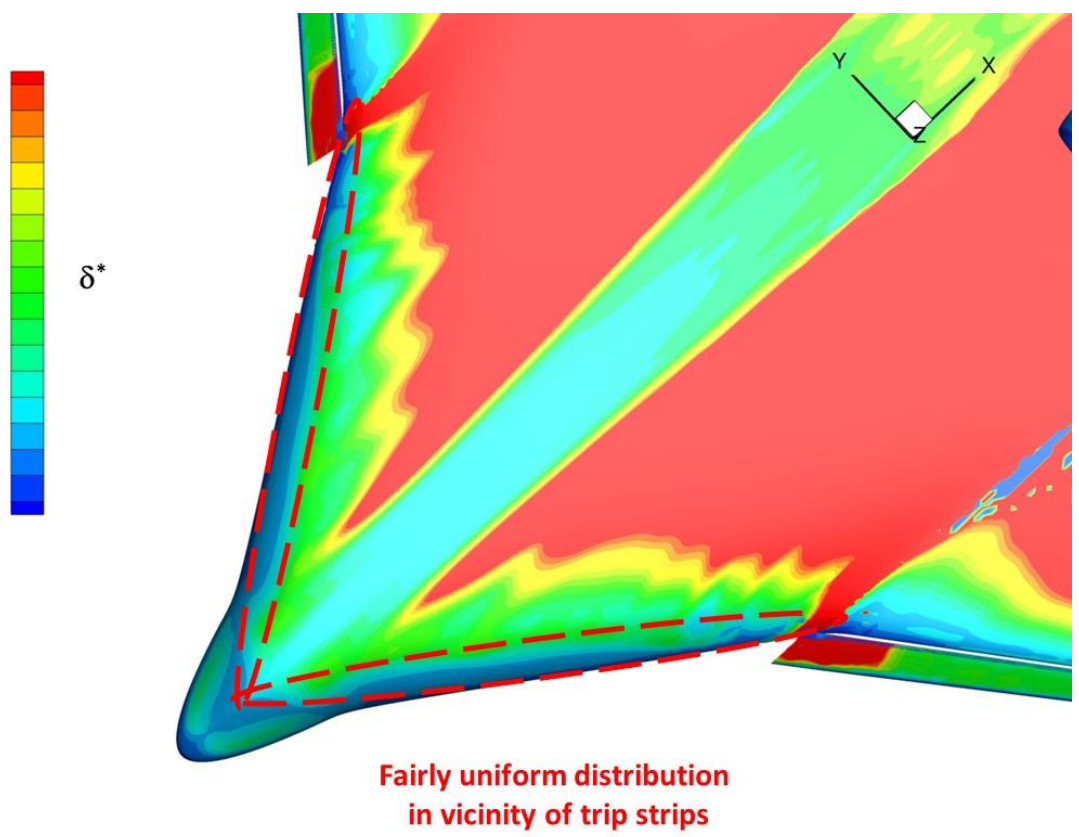

Figure 23. Upper surface displacement thickness, OVERFLOW/LM. $M=0.2, \boldsymbol{R}_{\text {cref }}=5.27 \times 10^{6}, \alpha_{2}<\alpha_{d}<\alpha_{3}$.

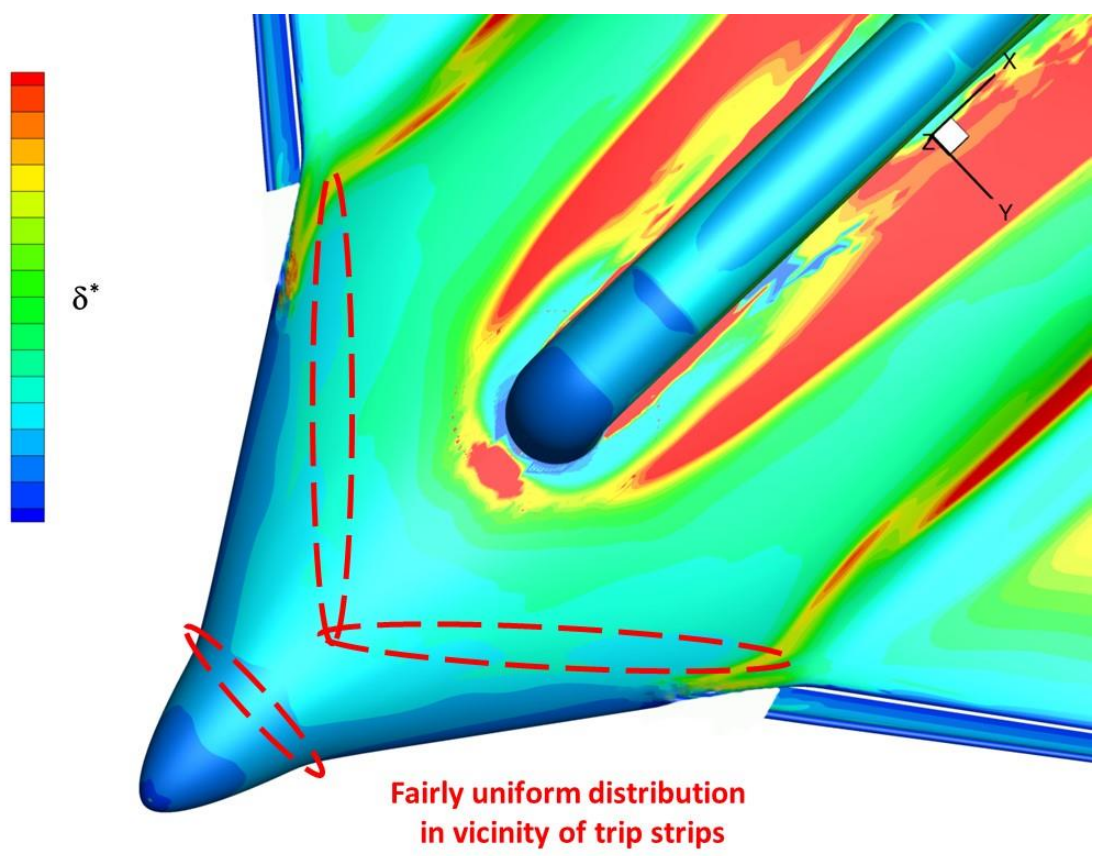

Figure 24. Lower surface displacement thickness, OVERFLOW/LM. $M=0.2, R e_{c r e f}=5.27 \times 10^{6}, \alpha_{2}<\alpha_{d}<\alpha_{3}$. 


\section{Final Trip Dot Patterns and Boundary-Layer Transition Verification}

The final trip dot patterns were applied to the HWB model and assessed early in the initial test entry at the LaRC 14- by 22-Foot Subsonic Tunnel. Infrared thermography was used with sufficient resolution to distinguish laminar and turbulent flows. Some examples of the final trip dot patterns, and details of test technique as well as the resultant verification measurements follow.

\section{A. Trip dot patterns}

Uniform size trip dots are available on linear strips (see Figure 25), and the piecewise linear boundary layer trip strips, with uniform trip dot size, as described in this paper were therefore practical to implement. The brand of trip dot strips used in this experiment were available in 20 different color-coded heights, between 0.0015 " and 0.017 " tall, and these discrete dot sizes were used in the final dot size selection from the hybrid traditional/CFD process described above. An average dot height could be found that (i) was suitable for each piecewise linear trip strip, and that (ii) would be effective for the majority of the angle-of-attack range of interest. In most cases, the dot height selection was biased toward a selected angle of attack within the full range. Regardless of height, all trip dots are 0.050 " in diameter with 0.10 " between dot centers.

Templates were fabricated to guide positioning of the trip strips on the wind tunnel model. An example is shown in Figure 26. Figure 26a shows the cad of a nose template used for some of the set points for the piecewise linear trip strips on the body. Figure 24b shows a lower-surface body template used for positioning the lower-surface centerline trip strip. Templates were used for all other set points of the piecewise linear trip strips for the various components of the wind tunnel model. These assured consistent placement of the strips throughout the ERA HWB low-speed wind tunnel test program.

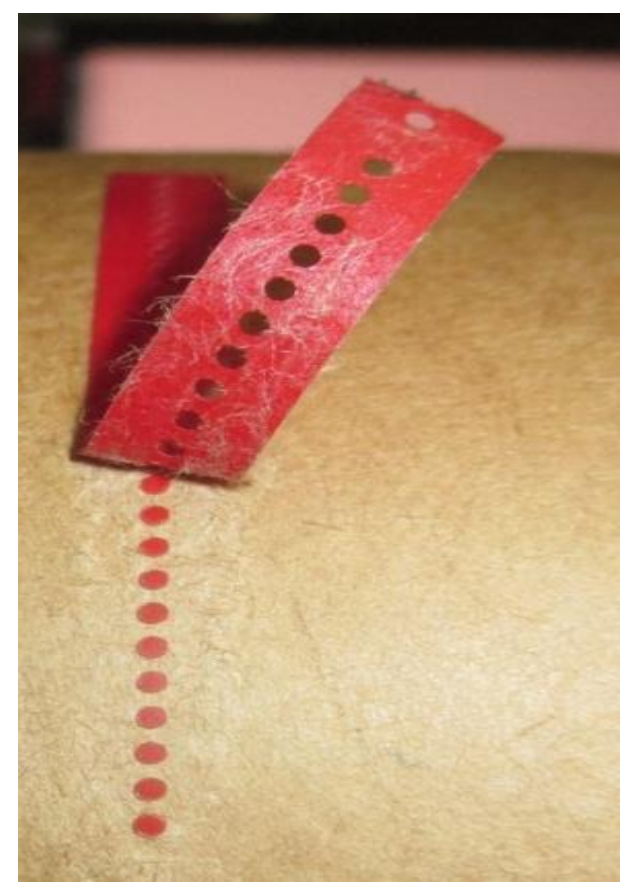

Figure 25. Prepackaged trip dot strip.

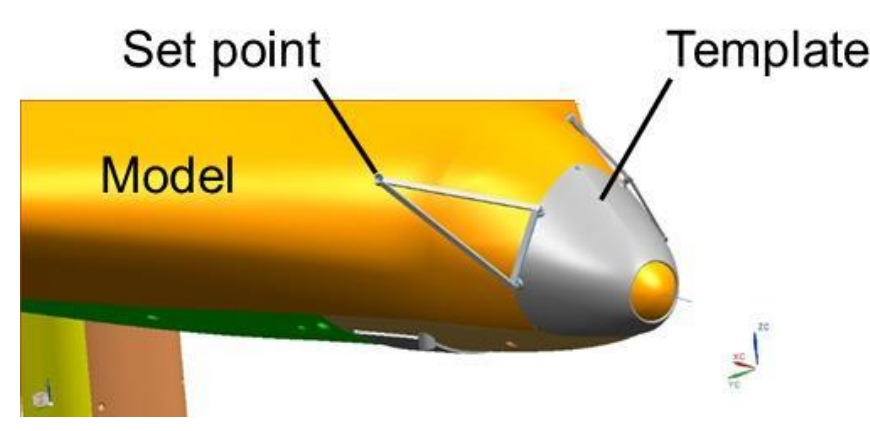

a) CAD display of a nose template.

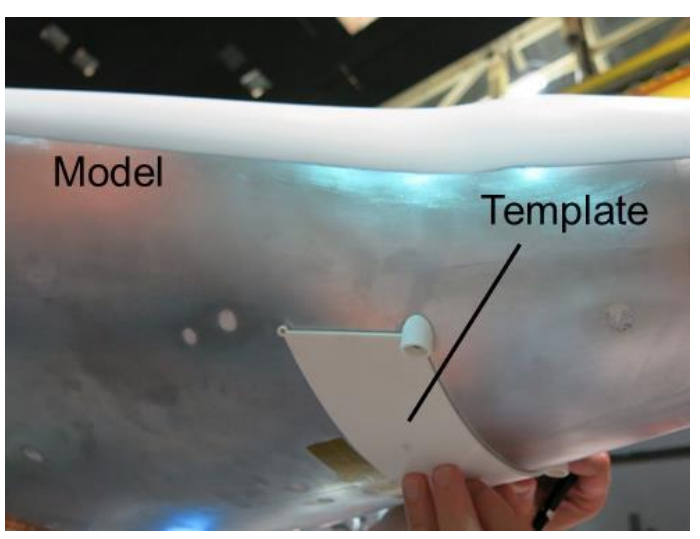

b) Lower-surface body template.

Figure 26. Transition strip positioning templates.

Some examples of trip-dot patterns on the 5.75\% ERA Hybrid-Wing-Body wind tunnel model are shown in Figure 27. In Figure 27a, the lower surface segmented trip strip pattern for the low-to-moderate angle-of-attack range can be seen on the model. Figure 27b shows a trip dot detail near the swept and blunt leading edge of the body on the lower surface, and is an example of the very uniform trip dot implementation that can be achieved with the trip strips that were used for this experiment. Figure $27 \mathrm{c}$ shows the trip dot ring that was applied near the nose of the model. 


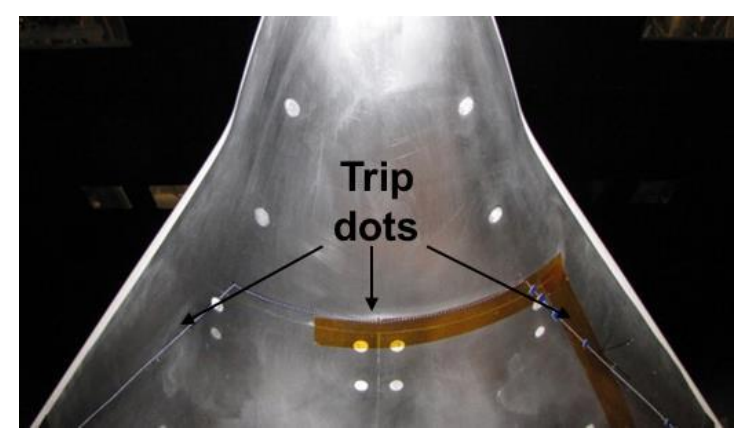

a) Body lower surface centerline region.

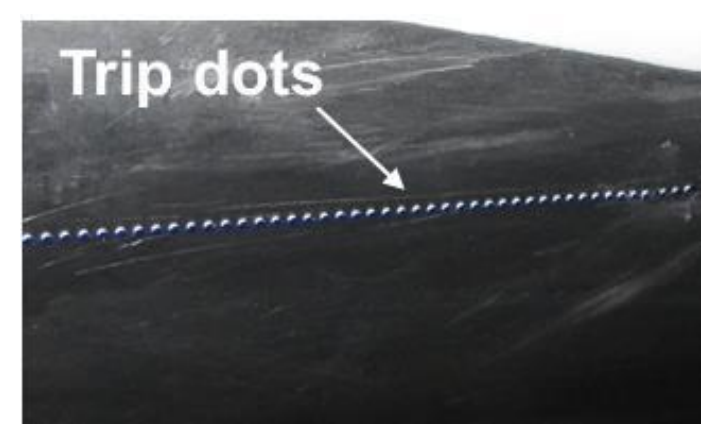

b) Body lower surface leading-edge detail.

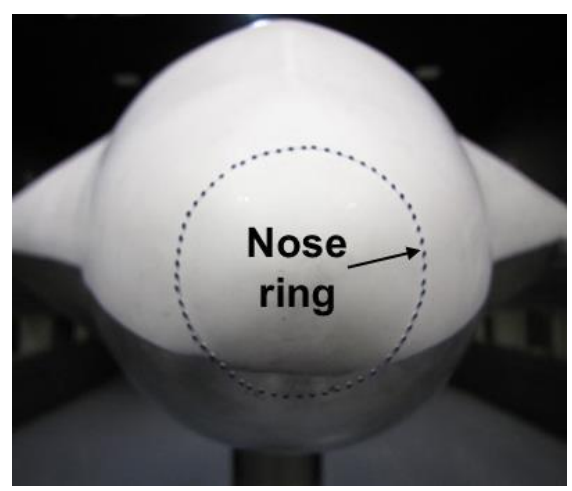

c) Nose-ring trip-dot strip.

Figure 27. Trip dot patterns on the HWB wind tunnel model.

\section{B. Verification test technique}

Infrared (IR) thermography was used to assess the effectiveness of the trip strips in transitioning the flow from a laminar to a turbulent state. Increased heat transfer from the free stream to the model occurs for a turbulent boundary layer, as compared to a laminar boundary layer, due to turbulent mixing. For the present interests, this would mean that a rather abrupt change in model surface temperature would occur at the location of an effective forced transition strip. The thermal detection needs in this case are essentially binary (cool temperatures for laminar flow, warmer temperatures for turbulent flow), and as such black and white imagery could suffice to detect the transition front.

For the HWB tests in the LaRC 14- by 22 Foot Subsonic Tunnel, a FLIR Systems SC6100 MWIR (Medium Wavelength Infrared) camera, controlled by a Windows laptop computer, was employed to collect imagery of the model in the vicinity of the trip strips and assess their effectiveness. This camera used a Cooled Indium Antimonide (InSb) Detector, had $640 \times 512$ resolution at 14 bits, and had an operating range of $-40 \operatorname{deg} F$ to $122 \operatorname{deg} F$. The camera was

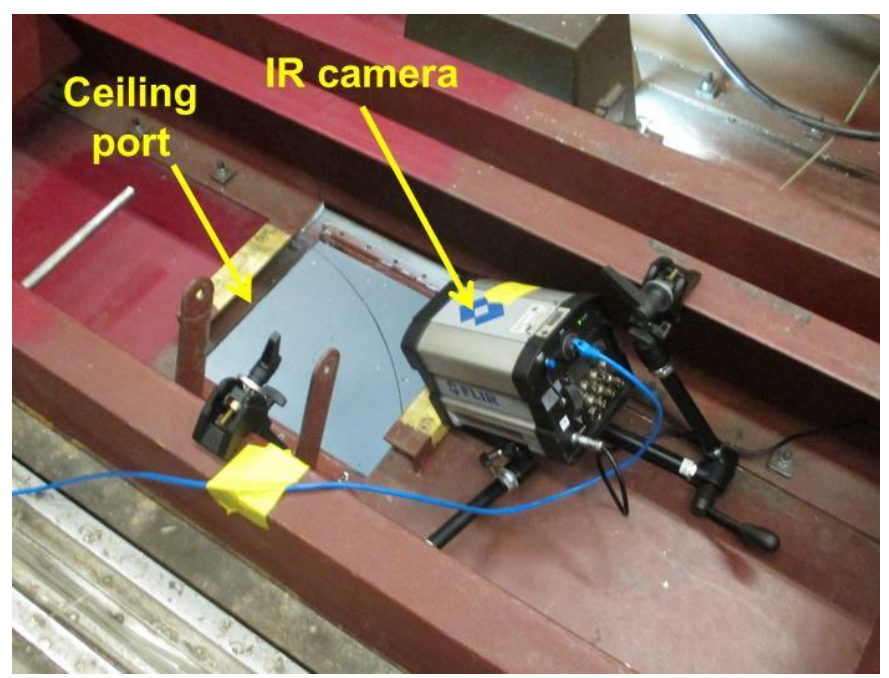

Figure 28. Infrared camera mounted in LaRC 14- by 22Foot Subsonic Tunnel. mounted in one of two locations: (i) the ceiling, which was used to assess transition on the wings and fuselage, and (ii) the side wall, which was used to assess transition along the lower surface of the fuselage and nacelle inlets. A photograph of the camera mounted in the ceiling of the LaRC 14- by 22-Foot Subsonic Tunnel is shown in Figure 28, and these two viewing locations are 
shown in Figure 29. The majority of the assessments were performed with the ceiling location. Since the IR camera does not detect thermal variations through normal glass or plastic, the camera was mounted such that it viewed the model through small apertures in the ceiling or side wall. The IR thermography runs were performed at the beginning of a day when the model would still be relatively cool and thus provide a greater contrast between the laminar and turbulent regions of the flow. To facilitate thermal viewing of the lower IR-reflective metallic surfaces of the model, a very thin layer of clear Krylon paint was applied to the lower surfaces of the model, eliminating the IR reflections, and providing a thin insulating layer.

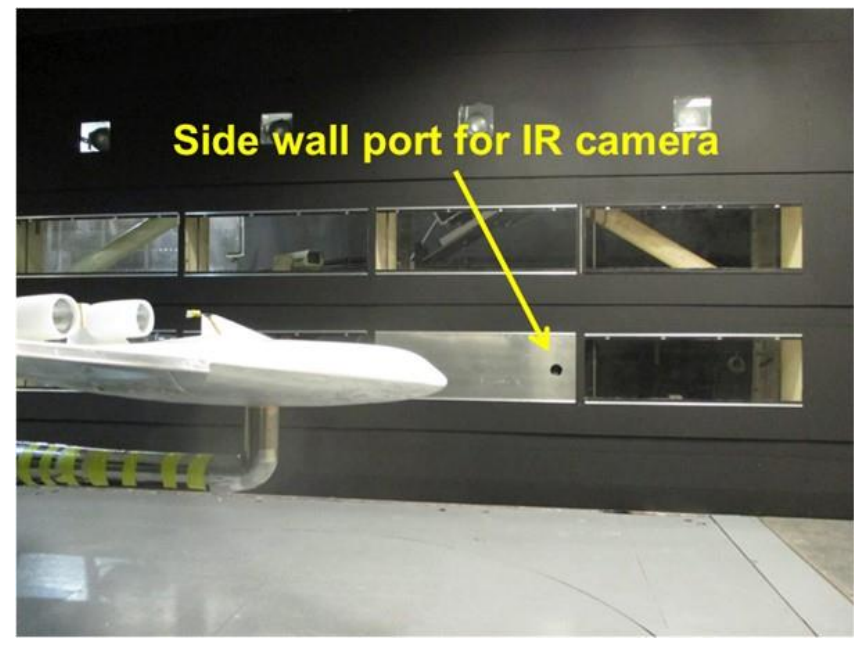

a) Side wall location.

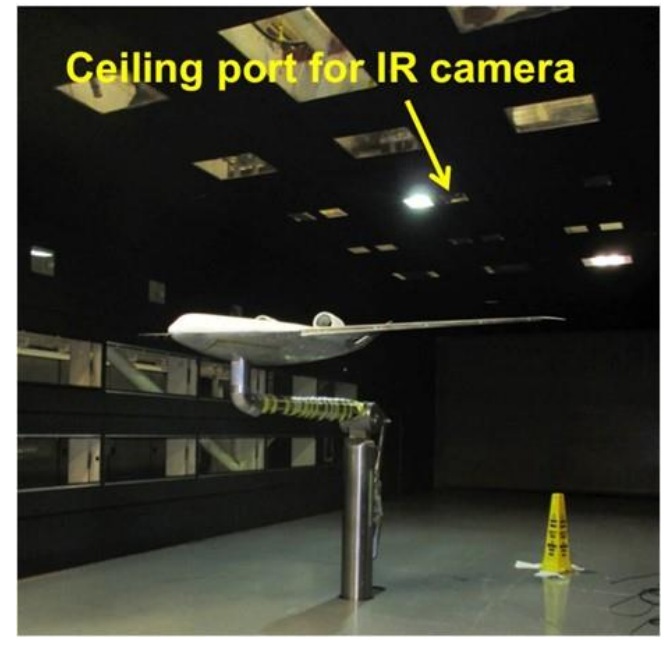

b) Ceiling location.

Figure 29. Infrared camera locations, LaRC 14- by 22-Foot Subsonic Tunnel.

\section{Boundary layer transition verification}

An orientation photograph for the infrared thermography is provided in Figure 30. Here the viewpoint is from the ceiling thermographic camera, and the view is looking down on the model and slightly upstream. Several components of the model are indicated, and the sharp demarcation between the dark body and the bright wing coincides with a seam between these two model components. The wing and body components were fabricated from different materials and this could account for the dissimilar thermal images at the seam between these two parts.

A close up of the thermographic images near the body leading-edge region is presented in Figure 31 for two runs, one with boundary layer trips off and the other with boundary layer trips on. Nominal test conditions were the same for these two runs. The tunnel air was warmer than the model, and the camera was set such that lighter colors represented warmer temperatures. In these circumstances, portions of the model with laminar flow will appear darker (less heat transfer) than portions of the model with turbulent flow (more heat transfer).

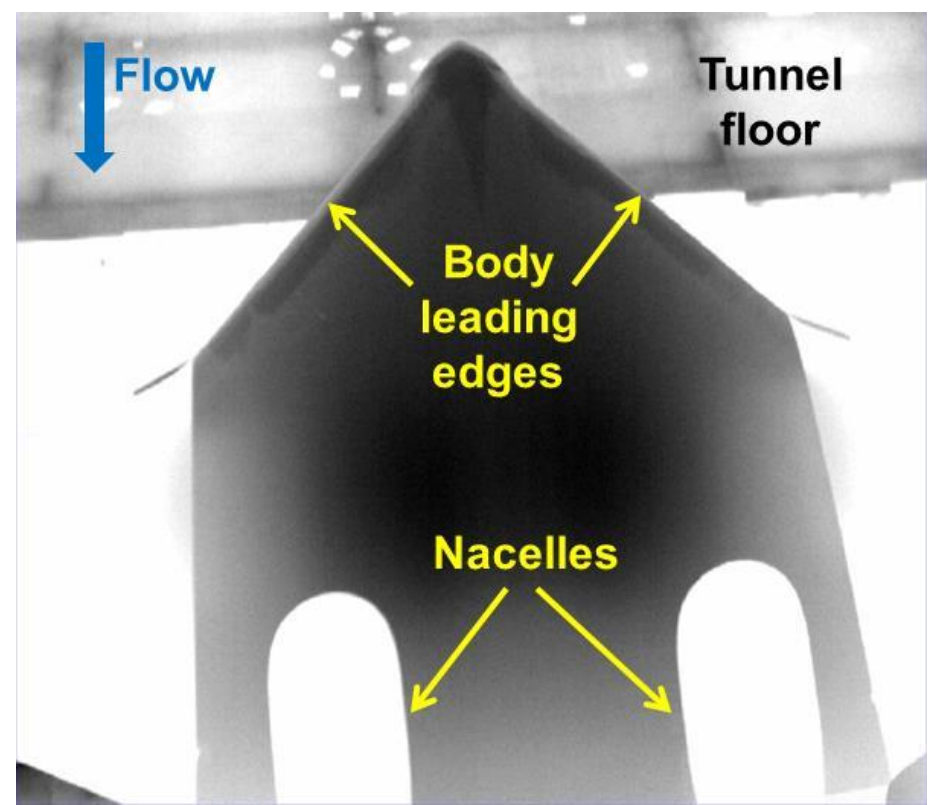

Figure 30. Thermographic image, ceiling perspective. $M=0.2, R e_{c r e f}=5.27 \times 10^{6}$, LaRC 14- by 22-Foot Subsonic Tunnel. 
Looking at the image with the boundary layer trips off (Figure 31a), the darker regions near the body leading edges and near the nose of the model correspond to laminar flow. The laminar region occurs for essentially all of the swept and blunt body leading edge. An abrupt but irregular transition to turbulent flow can also be observed.

For the image with the boundary layer trips on (Figure 31b), the dark regions near the body leading edges have been eliminated, and the trips appear to have successfully transitioned the flow. The region of laminar flow coming from the nose has been greatly reduced, but not eliminated. Some of the remaining laminar flow may be ahead of the trip location, and it is also possible that the trips here may have been undersized.

Both images show approximate lateral symmetry, with the exception of a turbulent wedge in Figure $30 \mathrm{~b}$ that is just left of the model centerline and near the nose. The turbulent wedge seems to emanate from a point forward and just out of view of the camera. Inspection of the model after this run revealed the remnants of a small bug that had impacted the model

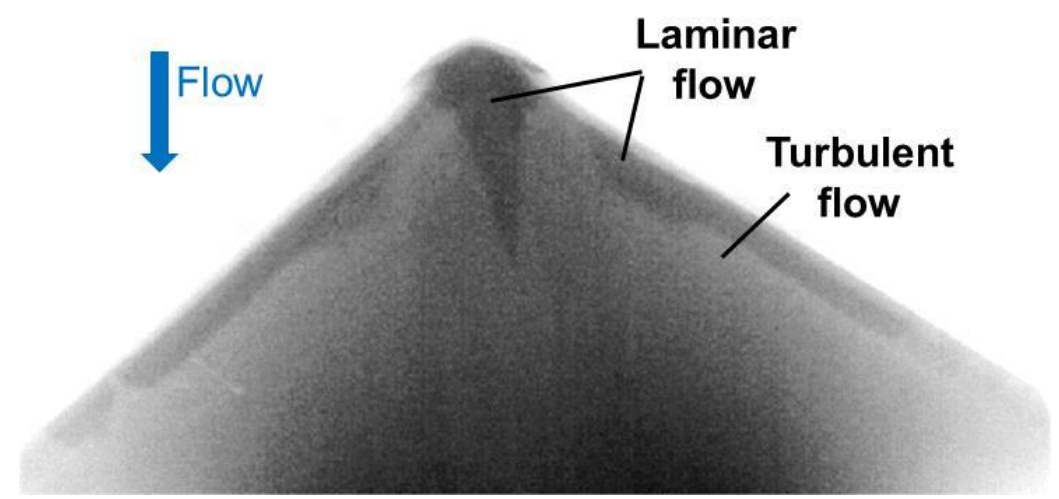

(a) Trips off.

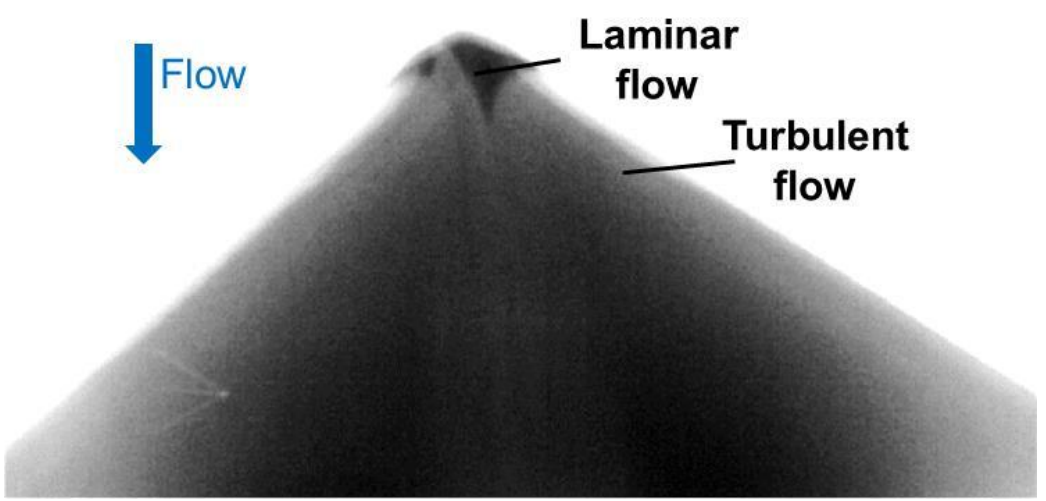

(b) Trips on.

Figure 31. Effect of trip dots on HWB upper surface thermal images. $M=0.2, R e_{c r e f}=5.27 \times 10^{6}$, LaRC 14- by 22-Foot Subsonic Tunnel. near the apparent origin of the turbulent wedge. The bug evidently tripped the otherwise laminar boundary layer, and this was captured on the thermographic image.

\section{Concluding Remarks}

A hybrid traditional/CFD-based test technique has been presented in this paper to guide placement and sizing of forced boundary-layer transition trips for the low-speed testing of an advanced Hybrid-Wing-Body wind tunnel model. The technique used CFD to estimate the boundary layer environment for the placement of trip strips and, in conjunction with traditional methods, to guide the trip dot sizing. Two trip patterns were developed, one for low-tomoderate angles of attack and the other for moderate-to-high angles of attack. The resultant boundary layer trip strips were practical to implement, and their effectiveness was verified with infrared thermographic photography.

The hybrid traditional/CFD-based technique outlined in this report was performed to guide placement and sizing of forced boundary-layer transition trips for one specific application, the low-speed aerodynamics of a HybridWing-Body configuration. However, the underlying principles for this process are all based on fundamental flow physics, and, as such, it is possible that the approach could be useful for other configurational applications.

\section{Acknowledgments}

This work was supported by the Environmentally Responsible Aviation (ERA) project, and the authors appreciate this support. The authors also would like to thank Ed Parlette for providing grids for the USM3D numerical simulations. 


\section{References}

${ }^{1}$ Frink, N. T., "Tetrahedral Unstructured Navier-Stokes Method for Turbulent Flow," AIAA Journal, Vol. 36, No. 11, Nov. 1998, pp. 1975-1982.

${ }^{2}$ Spalart, P.R. and Allmaras, S.R., "A One-Equation Turbulence Model for Aerodynamic Flows," Recherche Aerospatiale, No. 1, 1994, pp. 5-21.

${ }^{3}$ Braslow, A. L., Hicks, R. M., and Harris, R. V., Jr, "Use of Grit-Type Boundary-Layer-Transition Trips on Wind-Tunnel Models," NASA TN D-3579, Sep. 1966.

${ }^{4}$ Blackwell JA Jr: Preliminary Study of Effects of Reynolds Number and Boundary-Layer Transition Location on ShockInduced Separation. NASA TN D-5003, Jan. 1969.

${ }^{5}$ Gentry, G. L., Jr.; Quinto, P. F.; Gatlin, G. M.; and Applin, Z. T., "The Langley 14- by 22-Foot Subsonic Tunnel: Description, Flow Characteristics, and Guide for Users," NASA TP-3008, Sept. 1990.

${ }^{6}$ Hunt, R. and Sacco, J., “Activation and Operation of the National Full-Scale Aerodynamics Complex," AIAA Paper 20001076, Jan. 2000.

${ }^{7}$ Garcia, J. A., Melton, J., Schuh, M. J., James, K., Long, K., Vicroy, D. D., Deere, K. A., Luckring, J. M., Carter, M. B., Flamm, J. D., Stremel, P. M., Nikiado, B. E., and Childs, R. E., "NASA ERA Integrated CFD for Wind Tunnel Testing of Hybrid Wing-Body Configuration," AIAA 2016-0262, The 54 ${ }^{\text {th }}$ AIAA Aerospace Sciences Meeting, January 2016.

${ }^{8}$ Tramel, R. W., Nichols, R. H., and Buning, P. G., "Addition of Improved Shock-Capturing Schemes to OVERFLOW 2.1," AIAA 2009-3988, Jun. 2009.

${ }^{9}$ Langtry, R. B., and Menter, F. R., "Correlation-Based Transition Modeling for Unstructured Parallelized Computational Fluid Dynamics Codes,” AIAA Journal, Vol. 47, No. 12, Dec. 2009, pp. 2894-2906.

${ }^{10}$ Deere, K. A., Luckring, J. M., McMillin, S. N., Flamm, J. D., and Roman, D., "CFD Predictions for Transonic Performance of the ERA Hybrid Wing-Body Configuration", AIAA 2016-0266, 2016.

${ }^{11}$ Frink, N. T., Pirzadeh, S. Z., Parikh, P. C., Pandya, M. J., and Bhat, M. K., "The NASA Tetrahedral Unstructured Software System,” The Aeronautical Journal, Vol. 104, No. 1040, pp. 491-499, October 2000.

${ }^{12}$ Pandya, M. J., Abdol-Hamid, K. S., and Frink, N. T., "Enhancement of USM3D Unstructured Flow Solver for High-Speed High-Temperature Shear Flows," AIAA 2009-1329, The 47th AIAA Aerospace Sciences Meeting, January 5-8, 2009.

${ }^{13}$ Pao, S. P., “USMC6-TetrUSS Grid and Solution Cutter: A Brief Users' Guide," Version 4, NASA Langley Research Center, October 2008.

${ }^{14}$ Samareh, J., "GridTool: A Surface Modeling and Grid Generation Tool," Proceedings of the Workshop on Surface Modeling, Grid Generation, and Related Issues in CFD Solutions, NASA CP-3291, May 9-11, 1995.

${ }^{15}$ Pirzadeh, S., "Unstructured Viscous Grid Generation by Advancing-Layers Method," AIAA Journal, Vol. 32, No. 8, pp. 1735-1737, August 1994.

${ }^{16}$ Pirzadeh, S., "Structured Background Grids for Generation of Unstructured Grids by Advancing Front Method," AIAA Journal, Vol. 31, No. 2, pp. 257-265, February 1993.

${ }^{17}$ Suhs, N. E. Rogers, S. E., and Dietz, W. E. IPEGASUS 5: An Automated Pre-processor for Overset-Grid CFD," AIAA Paper 2002-3186, AIAA Fluid Dynamics Conference, June 2002, St. Louis, MO. 